\title{
Spatial signatures of anesthesia-induced burst-suppression differ between primates and rodents
}

\author{
Nikoloz Sirmpilatze*,1,2,3, Judith Mylius¹, Michael Ortiz-Rios ${ }^{1}$, Jürgen Baudewig1, \\ Jaakko Paasonen ${ }^{4}$, Daniel Golkowski5,7, Andreas Ranft6 ${ }^{6}$, Rüdiger Ilg5,8, Olli Gröhn", \\ Susann Boretius*,1,2,3,9
}

1. Functional Imaging Laboratory, German Primate Center - Leibniz Institute for Primate Research, Göttingen, 37077, Germany

2. Georg-August University of Göttingen, Göttingen, 37073, Germany

3. International Max Planck Research School for Neurosciences, Göttingen, 37075, Germany

4. A.I.V. Institute for Molecular Sciences, University of Eastern Finland, Kuopio, 70150, Finland

5. Department of Neurology, Klinikum Rechts der Isar der Technischen Universität München, Munich, 81675, Germany

6. Department of Anesthesiology and Intensive Care Medicine, Klinikum Rechts der Isar der Technischen Universität München, Munich, 81675, Germany

7. Department of Neurology, Heidelberg University Hospital, Heidelberg, 69120, Germany

8. Department of Neurology, Asklepios Stadtklinik Bad Tölz, Bad Tölz, 83646, Germany

9. Leibniz Science Campus Primate Cognition, Göttingen, 37077, Germany

${ }^{*}$ Correspondence:

- Nikoloz Sirmilatze (lead contact), nsirmpilatze@dpz.eu

- Susann Boretius (senior author), sboretius@dpz.eu 


\section{Abstract}

During deep anesthesia, the electroencephalographic (EEG) signal of the brain alternates between bursts of activity and periods of relative silence (suppressions). The origin of burst-suppression and its distribution across the brain remain matters of debate. In this work, we used functional magnetic resonance imaging (fMRI) to map the brain areas involved in anesthesia-induced burst-suppression across four mammalian species: humans, long-tailed macaques, common marmosets, and rats. At first, we determined the fMRI signatures of burst-suppression in human EEG-fMRI data. Applying this method to animal fMRI datasets, we found distinct burst-suppression signatures in all species. The burst-suppression maps revealed a marked inter-species difference: in rats the entire neocortex engaged in burst-suppression, while in primates most sensory areas were excluded-predominantly the primary visual cortex. We anticipate that the identified species-specific fMRI signatures and whole-brain maps will guide future targeted studies investigating the cellular and molecular mechanisms of burst-suppression in unconscious states.

\section{Keywords}

Anesthesia, burst-suppression, fMRI, isoflurane, sevoflurane, primate, rodent, macaque, marmoset, rat. 


\section{Introduction}

Despite the long-standing and successful practice of general anesthesia, its underlying mechanisms remain elusive. Most anesthetics lead to a dose-dependent transition from mild sedation to deep unconsciousness, accompanied by a progression of distinct electroencephalographic (EEG) patterns (Brown et al., 2011, 2010). This progression is well characterized for halogenated volatile agents, like isoflurane (Kroeger et al., 2013). Low isoflurane concentrations lead to high-amplitude low-frequency activity, whereas at higher concentrations, the ongoing activity is quasi-periodically interrupted by quiescent periods that can last from seconds to minutes. This alternation between bursts of activity and gaps of quiescence is termed burst-suppression (Swank, 1949; Swank and Watson, 1949). As anesthesia deepens, suppression periods become longer and ultimately culminate in complete electrical silence. The same sequence unfolds for many other anesthetics (Akrawi et al., 1996; Fleischmann et al., 2018) and even during deep hypothermia (Westover et al., 2015; Zhang et al., 2010). Burst-suppression also appears in comas of various etiologies, for which it holds diagnostic and prognostic value (Brenner, 1985; Brown et al., 2010; Cloostermans et al., 2012; Hofmeijer et al., 2014; Young, 2000). Clinically, burst-suppression is often used as the target EEG pattern for intended neuroprotection as well as pharmacological control of intracranial hypertension (An et al., 2015; Westover et al., 2015).

The presence of burst-suppression in a variety of unconscious conditions has led some researchers to propose that it represents a common low-order 'attractor' state-a hallmark of a profoundly inactive brain (Ching et al., 2012). Several models have focused on the temporal bistability (Bojak et al., 2015; Ching et al., 2012; Kroeger and Amzica, 2007; Liley and Walsh, 2013), while many open questions surround the spatial properties of bursts-namely, their origin and propagation across brain areas. Bursts have traditionally been viewed as globally synchronous events, based on their simultaneous occurrence across EEG electrodes (Clark and Rosner, 1973; Swank, 1949). However, recent findings from electrocorticogram in humans (Lewis et al., 2013) and widefield calcium imaging in rats (Ming et al., 2021), have challenged this view by demonstrating considerable spatial variation in burst origin and propagation. Furthermore, it is yet unclear whether burst-suppression is intrinsically cortical or is influenced by subcortical sources. According to one hypothesis, the cortex spontaneously falls into burst-suppression upon anatomical or functional disconnection from other brain areas. This view is supported by observations of burst-suppression in isolated neocortical slice preparations (Lukatch et al., 2005; Lukatch and MacIver, 1996), but contradicted by the ability of sensory stimuli to evoke bursts indistinguishable from the spontaneously-occurring ones (Hartikainen et al., 1995; Hudetz and Imas, 2007; Kroeger and Amzica, 2007; Land et al., 2012). 
Incorporating detailed spatial information into burst-suppression models could reduce the pool of possible mechanisms. Such information, in the form of 3D whole-brain maps, can be provided by blood-oxygen-level-dependent (BOLD) functional magnetic resonance imaging (fMRI, Logothetis and Pfeuffer, 2004; Ogawa et al., 1990). The two distinct phases of burst-suppression correspond to different energy consumption levels, which should-through neurovascular coupling-translate into noticeable changes in blood flow and oxygenation (Hillman, 2014). This premise has been confirmed in several species. In rats, a combination of EEG with optical imaging of cerebral blood flow revealed that bursts are coupled with strong hemodynamic responses (Liu et al., 2011). The same effect was demonstrated in macaques (Zhang et al., 2019) and humans (Golkowski et al., 2017) using simultaneous EEG-fMRI. These two studies have shown that the burst timing from EEG can be used for fMRI mapping of burst-suppression.

While the preservation of neurovascular coupling during burst-suppression holds promise for clinical research, it creates pitfalls for the increasingly popular animal fMRI studies. About 4/5 of such studies are conducted under anesthesia, most commonly with isoflurane (Mandino et al., 2020). Given that medium-to-high concentrations of isoflurane lead to burst-suppression (Kroeger et al., 2013), we hypothesized that many animal fMRI experiments have been conducted during this state. This assumption is supported by reports of global BOLD signal synchronization in isoflurane-anesthetized animals (Kalthoff et al., 2013; Liu et al., 2013; Paasonen et al., 2018), as is expected during burstsuppression (Zhang et al., 2019). Animal fMRI researchers typically try to avoid this confounding effect by using low concentrations of isoflurane (Liu et al., 2013), opting for different anesthetic regimes (Kalthoff et al., 2013; Paasonen et al., 2018), or applying global signal regression during fMRI preprocessing (Kalthoff et al., 2013; Liu et al., 2013; Zhang et al., 2019).

Importantly, burst-suppression is not a nuisance variable, but a biologically and clinically significant neural phenomenon. Here we show that fMRI data from anesthetized humans and animals can be exploited to map the spatial distribution of burst-suppression using a data-driven approach. Unlike previous studies, our strategy applies the same methodology across species, allowing us to examine whether specific brain regions are involved universally across mammals. To achieve this, we first implemented a simple heuristic algorithm for identifying the fMRI signatures of burst-suppression in a human EEG-fMRI dataset (Golkowski et al., 2017; Ranft et al., 2016). We then used this algorithm to detect instances of burst-suppression in fMRI data from anesthetized animals (macaques, marmosets, and rats), and constructed species-specific whole-brain maps of burst-suppression. These maps provide a detailed spatial account of burst-suppression in some of the most widely used animal models and reveal crucial differences between primates and rodents. The maps will serve as a guide for identifying promising target regions and facilitate translational research into the mechanisms of burst-suppression and its role in brain pathophysiology. 


\section{Results}

\section{fMRI signatures of EEG-defined burst-suppression in anesthetized humans}

Simultaneous EEG-fMRI recordings are ideal for constructing a whole-brain map of burst-suppression (Golkowski et al., 2017; Zhang et al., 2019): EEG supplies the timing of burst and suppression phases, which is convolved with a hemodynamic response function (HRF) and correlated with fMRI to produce voxel-wise statistical parametric maps. However, simultaneous EEG-fMRI recordings are challenging, particularly at high magnetic field strengths. Knowing the exact hemodynamic correlates of burst-suppression may circumvent the need for concurrent EEG, especially if these correlates are specific enough to serve as a unique fMRI signature.

To explore this approach, we revisited an EEG-fMRI dataset acquired in anesthetized human participants (Ranft et al., 2016), which contains task-free measurements at three different concentrations of the anesthetic sevoflurane: 3.9-4.6\% ('high'), 3\% ('intermediate') and 2\% ('low'). Importantly, this concentration range spans across EEG states, allowing us to examine how they differ in their fMRI patterns. We categorized the EEG recordings as one of two states-burst-suppression or continuous slow wave activity (uninterrupted by suppressions). The high sevoflurane concentration had been intentionally chosen in the initial study to induce burst-suppression (Ranft et al., 2016), and indeed 19/20 subjects exhibited the typical bistable EEG alternation at this concentration (Fig. 1A). The same applied to $4 / 20$ subjects at the intermediate concentration, albeit with shorter suppression phases. The remaining intermediate-concentration recordings as well as all low-concentration recordings showed uninterrupted slow wave EEG activity. These results are consistent with the known dose-dependency of burst-suppression (Pilge et al., 2014).

To examine potential effects of the two aforementioned EEG states on fMRI time series, we used the so-called 'grayplot' or 'carpet plot' - a 2D representation of fMRI data suited for detecting global signal fluctuations (Aquino et al., 2020; Power, 2017). The carpet plot is a heatmap of BOLD signal intensity, where each row represents a normalized (z-score) voxel time series. Upon examining carpet plots from fMRI runs with EEG-defined burst-suppression, we identified pronounced signal fluctuations that spanned almost the entire cortex (Fig. 1C). Conversely, fMRI runs with continuous slow wave EEG activity showed no such cortex-wide fluctuations (see Fig. S1A).

Next, we sought to quantify the exact correspondence between widespread BOLD fluctuations and EEG burst-suppression. We performed principal component analysis (PCA) on the 2D matrix of cortical voxel time series, extracted the first five temporal components, and correlated them with the voxel time series. In fMRI runs with cortex-wide fluctuations, the bistable pattern visible on the carpet plot was captured by one of the principal components (PCs) - in most cases the first PC (Fig. 1D). This 

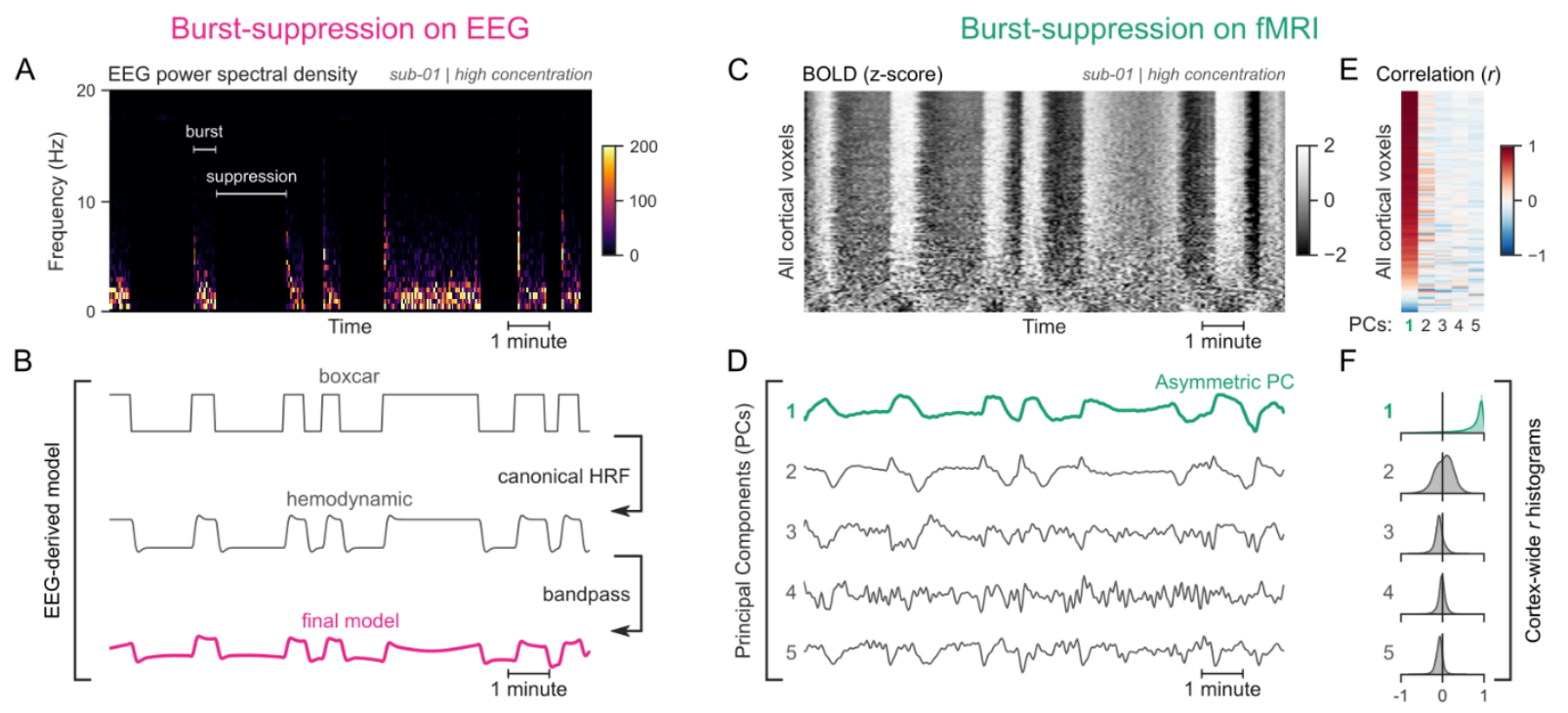

\section{Comparing $\mathrm{fMRI}$ to EEG}
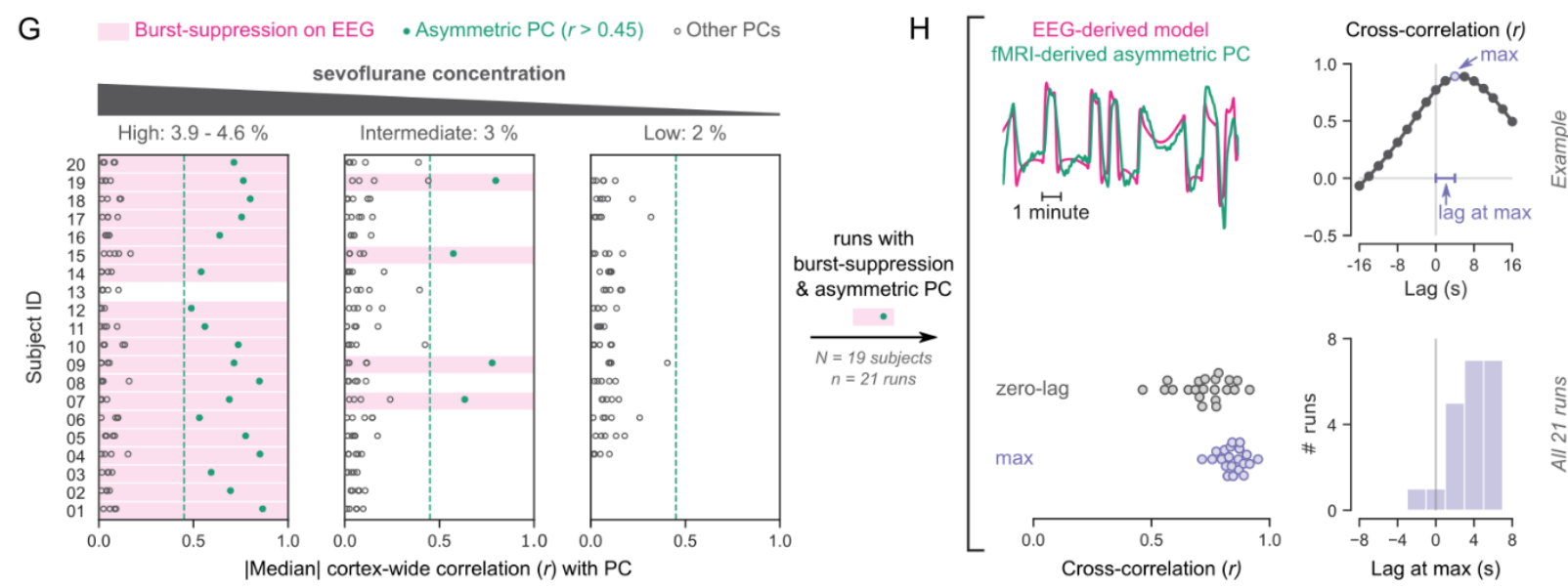

Figure 1. fMRI signatures of EEG-defined burst-suppression in anesthetized humans. (A) Burst and suppression phases are marked on an EEG spectrogram of a human participant under sevoflurane anesthesia. (B) To derive the hemodynamic model of burst-suppression, the above phases (boxcar function) are convolved with a hemodynamic response function (HRF) and bandpass filtered (0.005-0.12 Hz). (C) The cortical BOLD signal from the concurrent fMRI is represented as a 2D matrix (carpet plot). The rows (voxels) are ordered according to their correlation with the mean cortical signal. (D) The first five temporal principal components (PCs) of the shown matrix are plotted. Cortex-wide Pearson's correlation coefficients $(r)$ are shown for the first PCs, both as a heatmap (E) and as histograms for each PC (F). Taken together, panels C-F demonstrate that burst-suppression manifests as a widespread cortical BOLD signal fluctuation, captured by the first PC. This component (PC1), unlike the rest, is positively correlated with most cortical voxels and is referred to as an 'asymmetric PC'. (G). To demonstrate the correspondence between burst-suppression on EEG and asymmetric PCs on fMRI across the entire human dataset (20 participants, each with 2-3 EEG-fMRI runs at different sevoflurane concentrations), median cortex-wide $r$ values of the first five PCs are plotted as dots for each run. The runs that exhibit burst-suppression on EEG also have a single prominent asymmetric PC with median cortex-wide $r>0.45$ (except for subject 15 at high concentration). (H) For these runs, the cross-correlation between EEG-derived hemodynamic models and asymmetric PCs is shown. The cross-correlation at zero-lag, the maximum cross-correlation, and the time-lag at the maximum are extracted for each run (see example in top row) and plotted across all runs (bottom row). See also Fig. S1A. 
component was strongly correlated with most cortical voxel time series (Fig. 1E), shifting the histogram of Pearson's correlation coefficients $(r)$ away from zero-unlike the symmetric zerocentered histograms of other PCs (Fig. 1F). Due to this property, we will hereafter refer to such components as 'asymmetric' PCs and use the median of the cortex-wide $r$ values as a measure of asymmetry. The presence of asymmetric PCs proved to be a reliable signature of burst-suppression, as we demonstrate in Fig. $1 \mathrm{G}$ across subjects. The two EEG-defined states (burst-suppression vs continuous slow waves) were linearly separable based on the median $r$ value alone: every run with EEG burst-suppression had a single PC with median $r>0.45$, which was not the case for any of the runs with continuous slow waves. A single recording was an exception to this rule (subject 15 at high sevoflurane concentration, see Fig. 1G), exhibiting no asymmetric PC. The EEG of this recording showed near-constant suppression, interrupted by only one short burst.

So far, we have seen that burst-suppression is uniquely associated with an asymmetric PC that captures a widespread fluctuation in the cortical BOLD signal. Notably, this asymmetric PC closely follows the alternating burst-suppression pattern of EEG (Fig. 1A-D) and likely is a direct hemodynamic correlate. To verify this last observation, we compared each asymmetric PC with a neurovascular model derived from the concurrent EEG recording. For constructing the model, we convolved the EEG pattern (1 for burst, 0 for suppression) with a canonical HRF (Glover, 1999) and passed the resulting time series through the bandpass filter used for fMRI preprocessing $0.005-0.12$ Hz, see Fig. 1B). The BOLD signal of the asymmetric PCs was indeed strongly correlated with the EEGderived model (Pearson's $r=0.73 \pm 0.11$, mean \pm s.d.), but with a notable time-lag between the two. A cross-correlation analysis (Fig. $1 \mathrm{H}$ ) showed that the asymmetric PC was delayed by $3.71 \pm 2.22 \mathrm{~s}$ (mean \pm s.d.) compared to the neurovascular model. This lag can be attributed to a slowing of the $\mathrm{HRF}$ - an expected effect of anesthesia (Gao et al., 2017). We accounted for this lag by comparing the asymmetric PCs to the EEG-derived models at the time lags that maximized their cross-correlation and found that the correlation increased to $r=0.84 \pm 0.06$.

\section{Mapping burst-suppression in anesthetized humans without EEG}

Since asymmetric PCs are the hemodynamic correlates of burst-suppression, their spatial distribution should represent a whole-brain map of burst-suppression. The map should be equivalent to the one obtained via the existing approach-i.e. using the EEG-derived models as regressors in a general linear model (GLM, Golkowski et al., 2017). We verified this by performing two GLM analyses for each fMRI run with burst-suppression-one with the asymmetric PC and one with the EEG-derived model (without time lag correction). As expected, the spatial patterns of the resulting $Z$ statistical maps were nearly identical between the two analyses: neighborhood cross-correlation $r=0.96 \pm 0.03$ (Fig. $2 \mathrm{~A}$ ). 
A

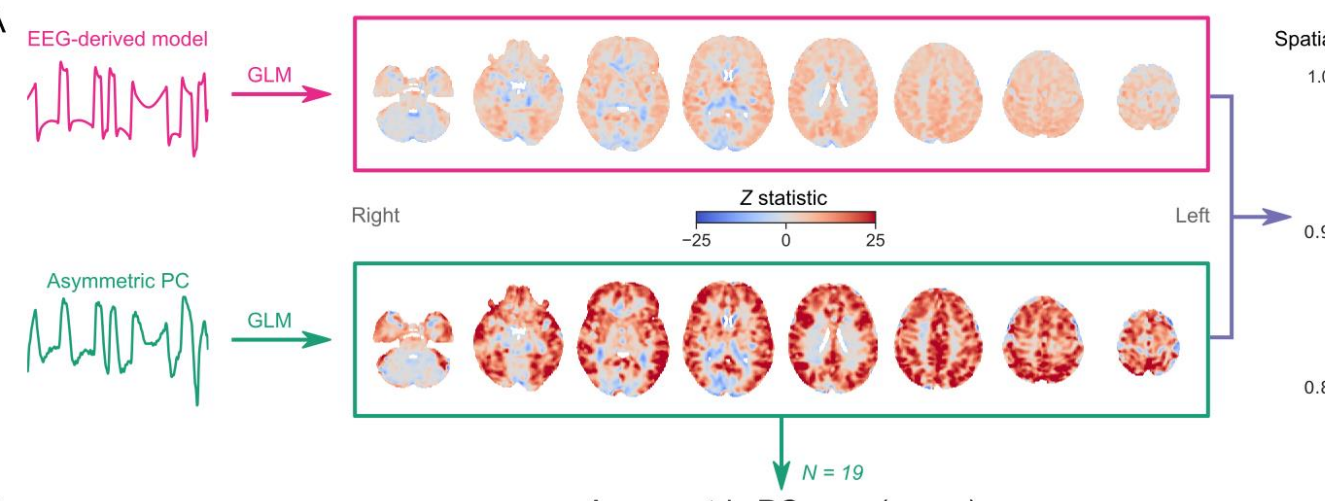

B

Asymmetric PC map (group)
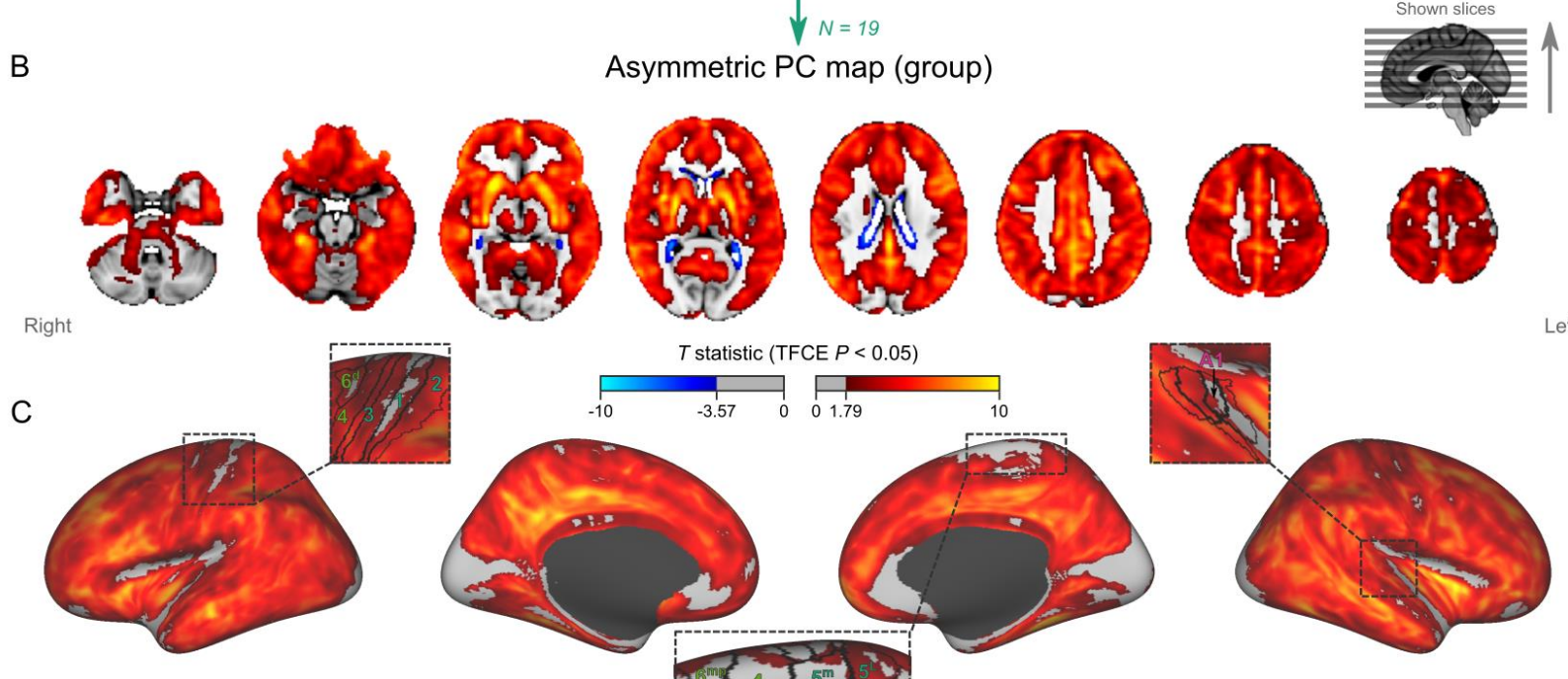

$\mathrm{D}$

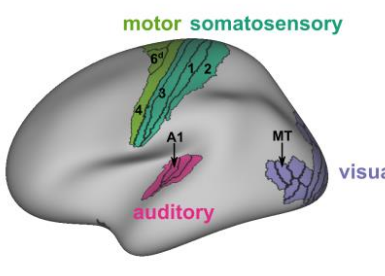

Left lateral

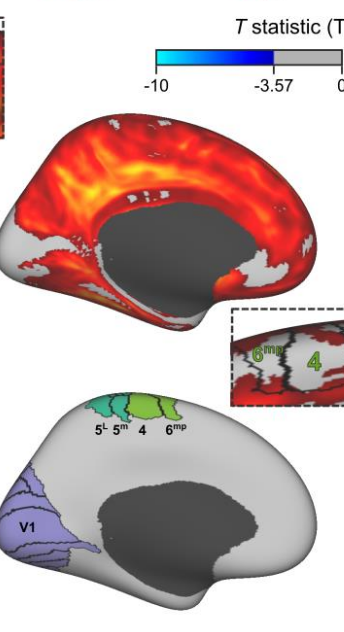

Left medial

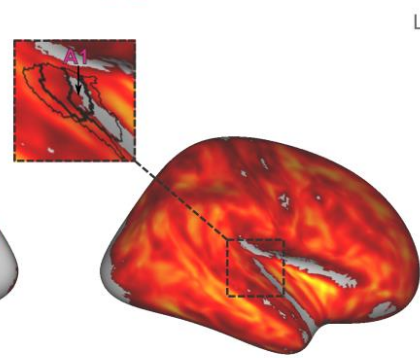

Figure 2. Mapping burst-suppression in anesthetized humans without EEG. (A) Maps of burst-suppression are computed via general linear model (GLM) analysis, using one of two regressors-either the EEG-derived hemodynamic model or the fMRI-derived asymmetric PC. The resulting $Z$ statistic maps for an example subject are shown here in the MNI152 template space. Neighborhood cross-correlation values between the two types of $Z$ statistic maps are plotted on the right, across all runs with asymmetric PCs ( $N=19$ subjects, $n=21$ EEG-fMRI runs). (B) The group burst-suppression map, computed via a second-level analysis of the single-subject asymmetric PC GLMs, is shown here overlaid on the MNI152 volumetric template. The group statistics were carried out with FSL randomise; the resulting $T$ statistic maps were thresholded using Threshold-Free Cluster Enhancement (TFCE) and a corrected $P<0.05$. (C) The same group map is shown in fsaverage surface space. Non-cortical areas on the medial surface are shown in dark grey. (D) The locations of several sensory and motor cortical areas, based on the HCP multimodal parcellation, are indicated on the surface: primary motor (area 4), premotor (areas $6 \mathrm{~d}$ and $6 \mathrm{mp}$ ), primary somatosensory (areas 3a-b, 1,2), higher somatosensory (areas $5 \mathrm{~m}$ and 5L), primary auditory, higher auditory (medial and lateral belt, parabelt), primary visual, and higher-order visual (V2, V3, V3A, V3B, V4, V4t, V6, V6A, V7, V8, MT, MST, and lateral occipital areas 1-3). See also Fig. S4A. 
To map the spatial distribution of burst-suppression across subjects, we performed a second-level group GLM using the asymmetric PCs as regressors. The group statistics were carried out in the MNI152 template space (Grabner et al., 2006) with the FSL (FMRIB's Software Library) tool randomise (Winkler et al., 2014). The resulting $T$ statistic maps were thresholded using ThresholdFree Cluster Enhancement (TFCE, Smith and Nichols, 2009) and a corrected $P<0.05$ (Fig. 2B). The group map revealed significant correlation with asymmetric PCs (burst-suppression) in the striatum and across most of the cortex, with the prominent exception of occipital areas in and around the calcarine sulcus - the location of primary visual cortex (V1). In addition to V1, several other cortical patches were non-correlated with burst-suppression. We visualized these on an inflated representation of the cortical surface (Fig. 2C-D). Some of them overlapped with primary cortices: somatosensory and motor areas around the central sulcus, and auditory areas on the dorsal bank of superior temporal gyrus-including Heschl's gyrus (Fig. 2D). Additional inactive patches included the subcallosal cortex (subgenual cingulate), the parahippocampal gyrus, and the border between the insula and the frontoparietal operculum. Fig. 4A provides a closer look at subcortical structures. Anterior and midline parts of the thalamus were significantly correlated with burst-suppression, whereas posterior thalamic nuclei, the cerebellar cortex, the hippocampus, and the amygdala were, for the most part, not engaged.

Intriguingly, we observed significant anticorrelation with burst-suppression at the ventricular borders. After a closer examination of this effect (see Fig. S2), we believe it relates to a cycle of ventricular shrinkage (during bursts) and expansion (during suppressions). The widespread hemodynamic fluctuations during burst-suppression may exert a 'pumping' effect on the ventriclesan explanation consistent with recent reports of cerebrospinal fluid oscillations during sleep (Fultz et al., 2019).

\section{Nonhuman primates exhibit human homologous $\mathrm{FMRI}$ signatures of burst-suppression}

Having identified an fMRI signature of burst-suppression in the human data, we searched for similar signatures in nonhuman primate fMRI data. We first evaluated an fMRI dataset of female long-tailed macaques (Macaca fascicularis), acquired at $3 \mathrm{~T}$. Macaques are phylogenetically close to humans, have a long tradition of being used for neuroscientific research, and account for most fMRI studies in nonhuman primates (Mandino et al., 2020; Milham et al., 2020). The 13 macaques included in our study were anesthetized with isoflurane (concentration range 0.95-1.5\%) and mechanically ventilated. For each macaque, one or two fMRI runs (each 10-20 min in duration) were acquired within a single imaging session. 
A

$\overline{0}$
0
0
0
$\frac{0}{0}$
B
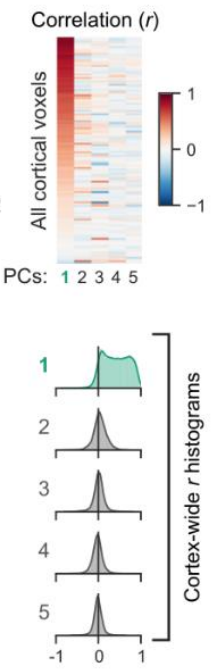

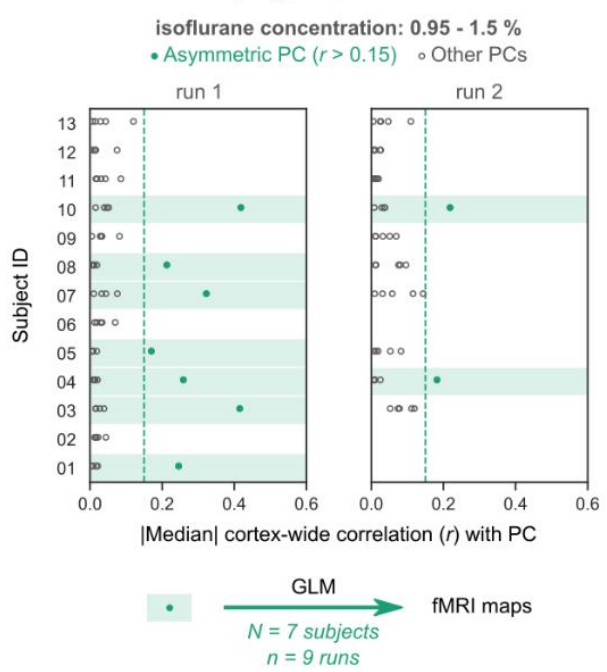

C
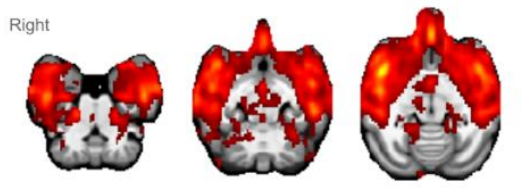

$T$ statistic (TFCE $P<0.05$ )

D

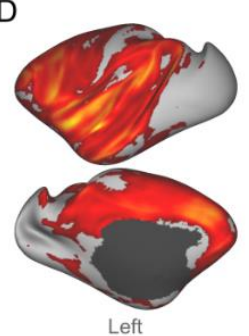

$\begin{array}{ll}{ }_{0} & 2.16\end{array}$
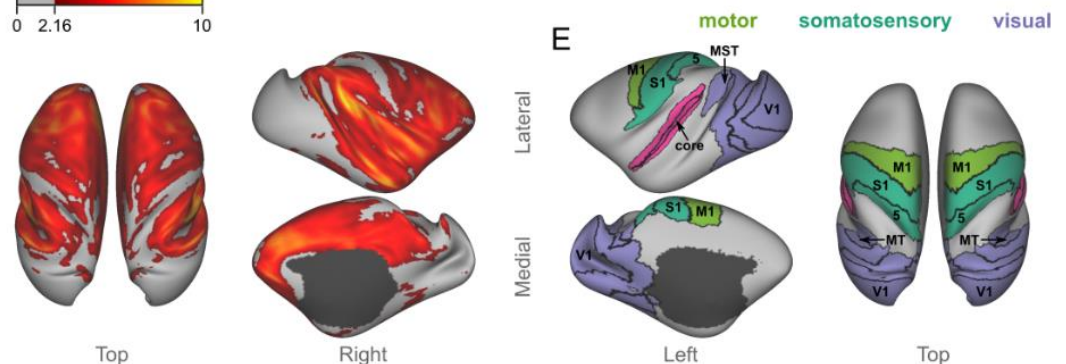

Top
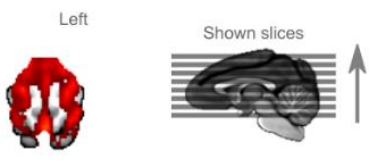

Figure 3. Macaques exhibit human homologous fMRI signatures of burst-suppression. (A) The cortical BOLD fMRI signal of a long-tailed macaque during isoflurane anesthesia is represented as a carpet plot. The rows (voxels) are ordered according to their correlation with the mean cortical signal. The first five temporal principal components (PCs) of the signal are plotted below. The Pearson's correlation coefficients $(r)$ between the PCs and all cortical voxels are represented both as a heatmap and as histograms for each PC. The first PC captures the widespread fluctuation visible on the carpet plot and has an asymmetric $r$ histogram. (B) Cortexwide median $r$ values for the first five PCs are plotted as dots across the entire macaque dataset. The fMRI runs with a single prominent asymmetric PC ( $r>0.15$, highlighted in green) are selected for general linear model (GLM) analysis. (C) The group asymmetric PC map, computed via a second-level analysis of single-subject GLMs, is shown here overlaid on a study-specific volumetric template. The group statistics were carried out with FSL randomise; the resulting $T$ statistic maps were thresholded using Threshold-Free Cluster Enhancement (TFCE) and a corrected $P<0.05$. (D) The same group map is shown on a cortical surface representation of the template. Non-cortical areas on the medial surface are shown in dark grey. (E) The locations of several sensory and motor cortical areas, based on the Cortical Hierarchical Atlas of the Rhesus Macaque (CHARM), are indicated on the surface: primary visual (V1), higher-order visual (V2, V3, V4, V6, MT, MST, FST), primary somatosensory (S1), higher somatosensory (area 5), primary motor (M1), and auditory cortices (auditory core, belt and parabelt). See also Fig. S1B and Fig. S4B. 
We analyzed each fMRI run using the same approach as for human fMRI data-carpet plots and PCA. Several fMRI runs clearly exhibited the burst-suppression signature: a widespread bistable fluctuation on the carpet plot that was always captured by an asymmetric PC (see example in Fig. 3A, counterexample in Fig. S1B). Fig. 3B shows the cortex-wide median $r$ value for the first five PCs across all fMRI runs. We selected nine runs (from 7/13 subjects) with a single prominent asymmetric PC (median $r>0.15$ ) and used these PCs as regressors for GLM. The group statistical map (Fig. 3C-E) exhibited striking homology with the human burst-suppression map. Significantly correlated areas included the striatum and most of the cortex, with the following exceptions: V1, extrastriate visual areas, parts of somatosensory and motor cortices on either side of the central sulcus, the subcallosal cortex and the parahippocampal gyrus. One notable difference to the human map was the auditory cortex, the entirety of which was among the positively correlated areas. The cerebellar cortex was not correlated with burst-suppression, while the thalamus, hippocampus, and amygdala were partly involved (Fig 4B). No significant anti-correlations were found around the ventricles or elsewhere.

\section{A. Human}
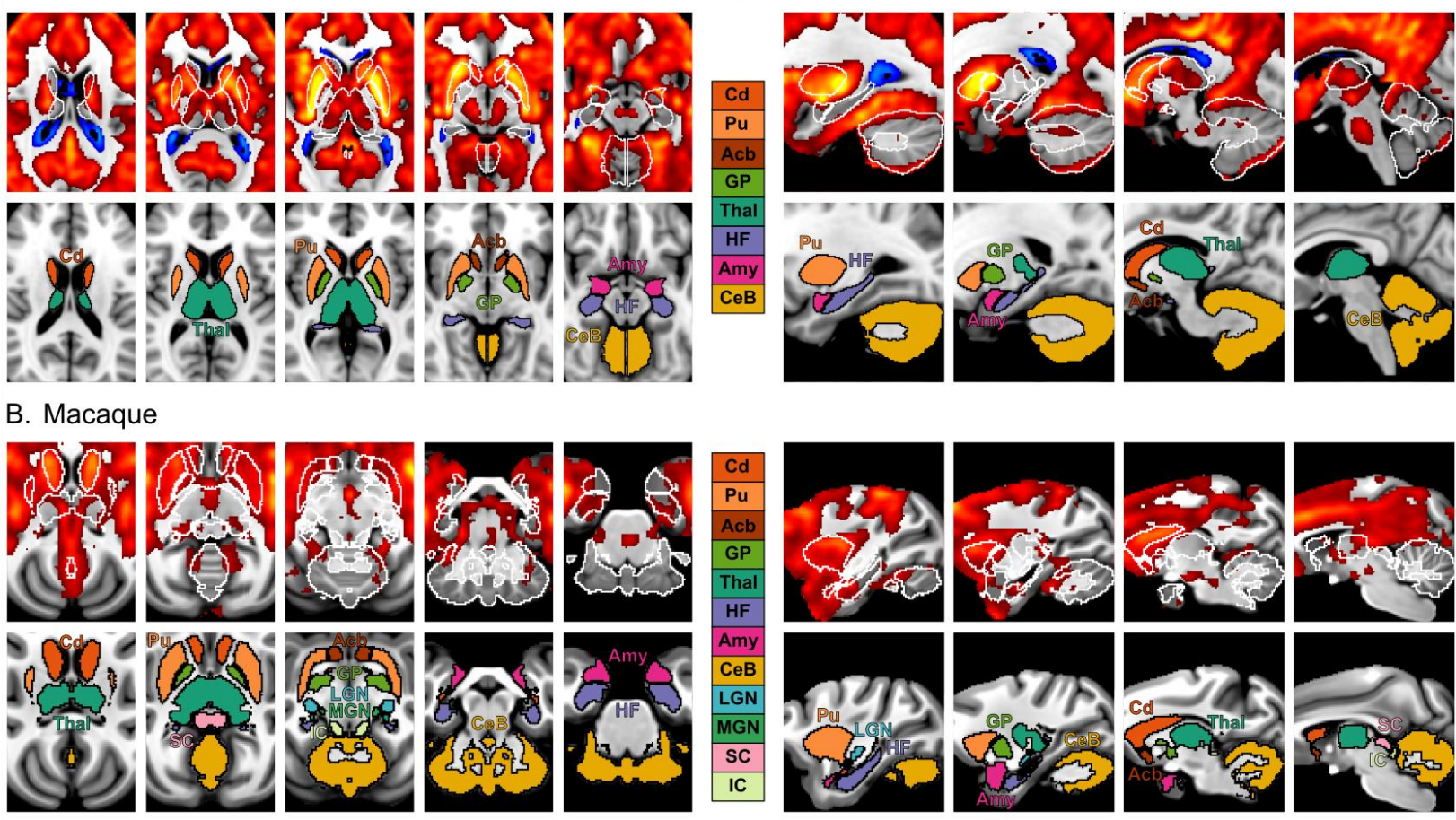

Right
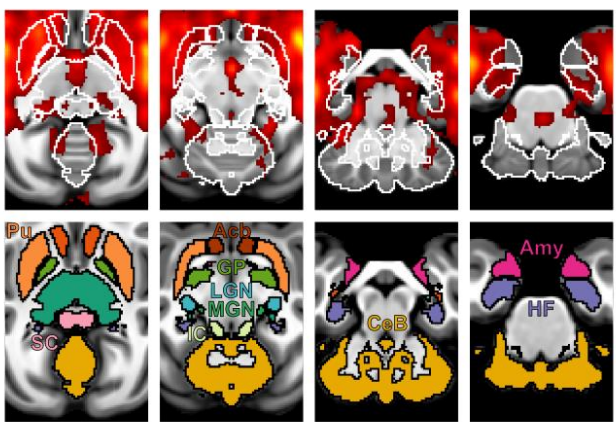

$\stackrel{\text { Axial slices }}{\longrightarrow}$ Inferior
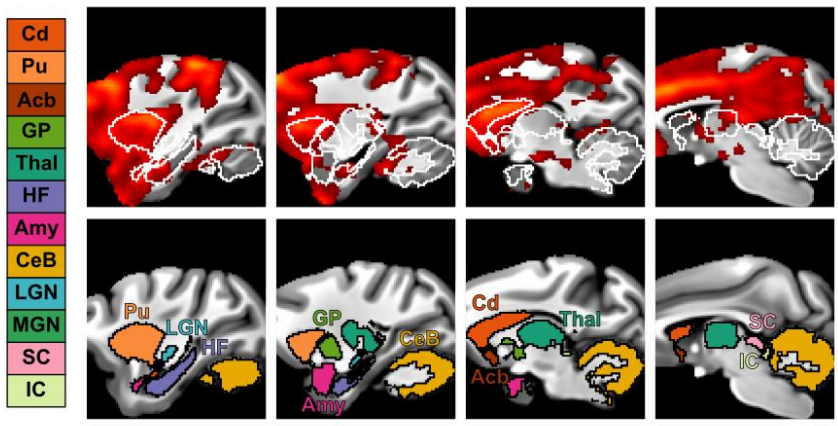

Anterior

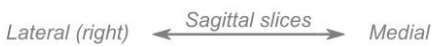

Posterior

Figure 4. Subcortical structures during burst-suppression in humans and macaques. (A) Group statistic map showing the spatial distribution of asymmetric PCs in humans (same as in Fig. 2B). The views are adjusted to focus on subcortical structures, on axial and sagittal slices. (B) The corresponding map is shown for macaques (same as in Fig. 3C). For each species, the bottom row shows the location of major subcortical structures, defined based on the human Harvard-Oxford subcortical structural atlas and the Subcortical Atlas of the Rhesus Macaque (SARM). Cd: caudate; Pu: putamen; Acb: accumbens; GP: globus pallidus; Thal: thalamus; HF: hippocampal formation; Amy: amygdala; CeB: cerebellum; LGN: lateral geniculate nucleus; MGN: medial geniculate nucleus; SC: superior colliculus; IC: inferior colliculus. 
A

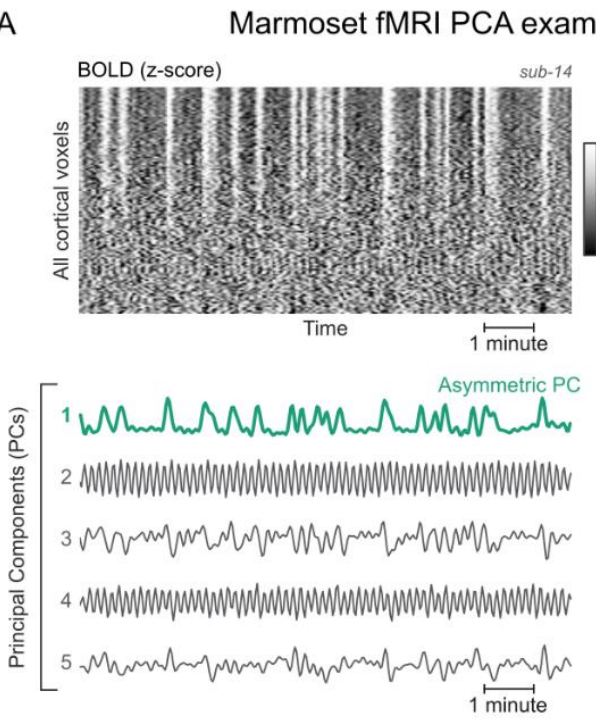

B
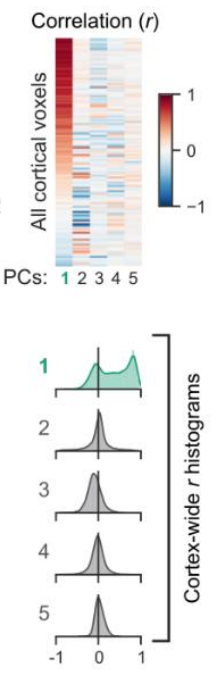

Identifying asymmetric PCs

isoflurane concentration: $0.6-1.1 \%$

-Asymmetric PC $(r>0.22) \quad \circ$ Other PCs

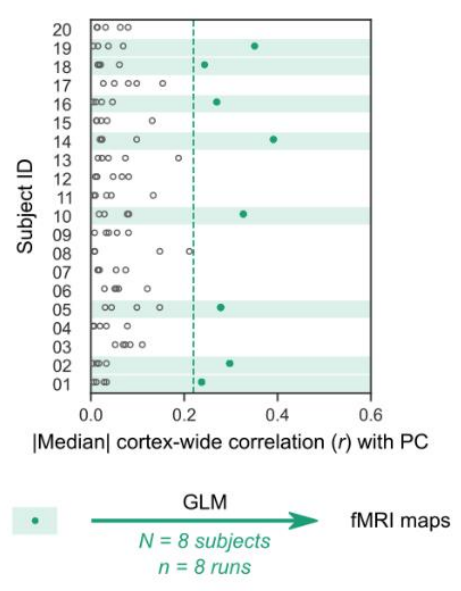

C

Asymmetric PC map (group)
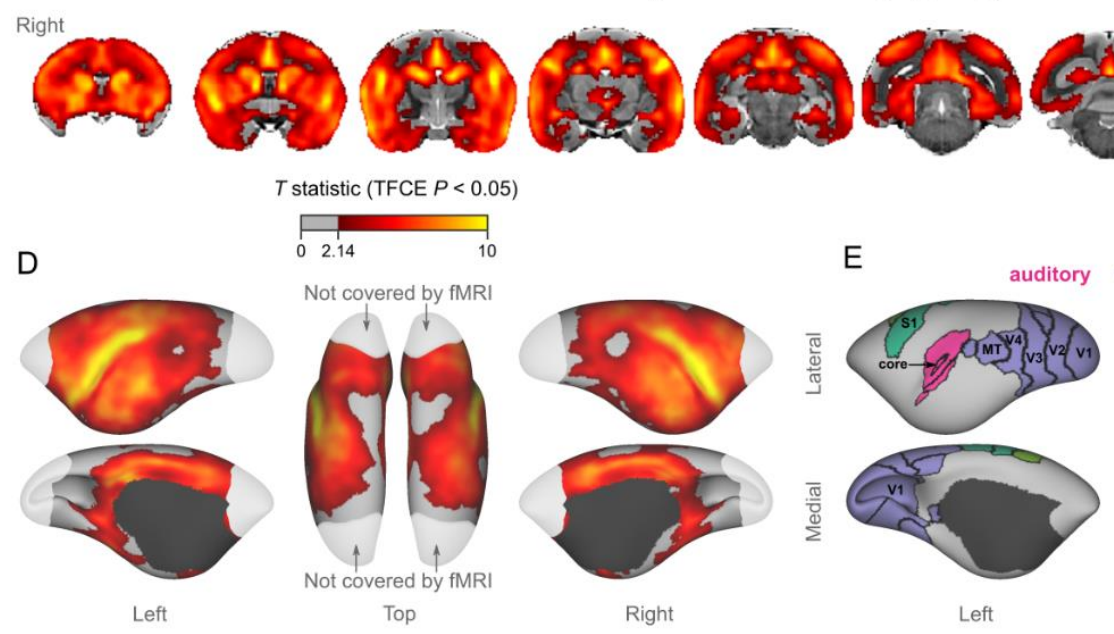

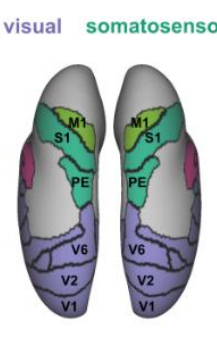

Top

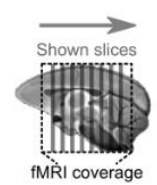

fMRें coverage
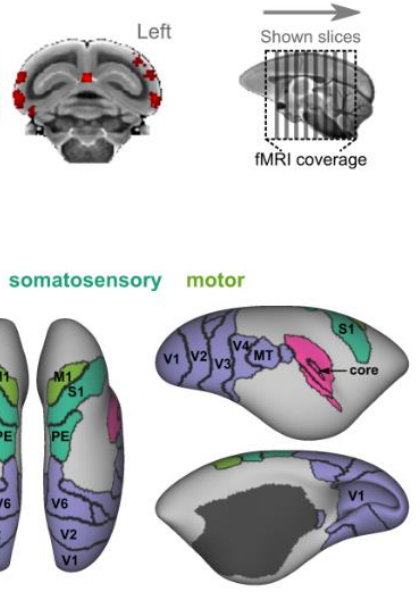

Right

Figure 5. Marmosets exhibit human homologous fMRI signatures of burst-suppression. (A) The cortical BOLD fMRI signal of a common marmoset during isoflurane anesthesia is represented as a carpet plot, with rows (voxels) ordered according to their correlation with the mean cortical signal. The first five temporal principal components (PCs) of the signal are plotted below. The Pearson's correlation coefficients $(r)$ between the PCs and all cortical voxels are represented both as a heatmap and as histograms for each PC. The first PC captures the widespread fluctuation visible on the carpet plot and has an asymmetric $r$ histogram. (B) Cortexwide median $r$ values for the first five PCs are plotted as dots across the entire marmoset dataset. The fMRI runs with a single prominent asymmetric PC $(r>0.22$, highlighted in green) are selected for general linear model (GLM) analysis. (C) The group asymmetric PC map, computed via a second-level analysis of single-subject GLMs, is shown here overlaid on the NIH v3.0 population template (T2-weighted image). The group statistics were carried out with FSL randomise; the resulting $T$ statistic maps were thresholded using Threshold-Free Cluster Enhancement (TFCE) and a corrected $P<0.05$. (D) The same group map is shown on a cortical surface representation of the template. Non-cortical areas on the medial surface are shown in dark grey. Areas not covered by the fMRI imaging volume are overlaid with white transparency. (E) The locations of several sensory and motor cortical areas, according to the NIH MRI-based cortical parcellation, are indicated on the surface: primary visual (V1), higher-order visual (V2, V3, V4, V6, MT, MST, Brodmann area 19), primary somatosensory (S1), higher somatosensory (area PE), primary motor (M1), and auditory cortices (auditory core, belt, parabelt, and superior temporal rostral area). See also Fig. S1C and Fig. S4C. 
We next aimed to reproduce our findings in the common marmoset (Callithrix jacchus). The layout of areas in the marmoset brain closely resembles that of other primates (Liu et al., 2018), including macaques and humans. Yet, the marmosets' body size is comparable to rats, and they fit into highfield small animal MRI systems that are routinely used for imaging rodents. These properties make the marmoset an ideal model for bridging neuroimaging results between larger primates and rodents. For this study we used fMRI data from 20 common marmosets (10/20 females), imaged at 9.4 T. The animals were anesthetized with isoflurane (concentration range 0.6-1.1\%) and mechanically ventilated. One fMRI run (10 min in duration) was acquired per animal.

Analyzing the data as described for humans and macaques, we discovered a subset of marmoset fMRI runs that exhibited the burst-suppression signature (see example in Fig. 5A, counterexample in Fig. S1C). We selected $8 / 20$ subjects with a single prominent asymmetric PC (median cortex-wide $r>0.22$ ) and used these PCs as GLM regressors (Fig. 5B). The group statistical map was overlaid on the NIH population template (Liu et al., 2021). The resulting map (Fig. 5C-E) was functionally homologous to the macaque map. Positively correlated areas comprised the striatum and a large part of the cortexincluding auditory areas, but excluding V1, extrastriate visual areas, parts of somatosensory and motor cortices, and most of the parahippocampal gyrus. The cerebellar cortex and the thalamus (except for a small anterior part) were not correlated with burst-suppression, but parts of the hippocampus and the amygdala were (Fig. 7A). No significant anti-correlations were found.

\section{Rats exhibit pancortical fMRI signatures of burst-suppression}

Finally, we asked how our observations translate to rodents, specifically rats-the most popular animal model in preclinical fMRI (Mandino et al., 2020). Several studies have identified burstsuppression in electrophysiological recordings of rats anesthetized with isoflurane, mostly at concentrations between 1.3-2\% (Detsch et al., 2002; Hudetz and Imas, 2007; Liu et al., 2011; Masamoto et al., 2009; Stenroos et al., 2021). One study described the potential fMRI correlate of burst-suppression in rats: widespread BOLD synchrony across the cortex and the striatum (Liu et al., 2011). Therefore, we anticipated at which isoflurane concentrations burst-suppression might occur, and how the maps might look like. To replicate these findings, we obtained rat fMRI data covering the concentration range between $1.5-2.5 \%$ isoflurane. The animals (11 adult female Wistar rats) were mechanically ventilated and imaged at $9.4 \mathrm{~T}$. We first acquired three fMRI runs at $2 \%$ isofluraneeach covering $12 \mathrm{~min}$, and then switched to either a lower (1.5\%, in 6/11 rats) or a higher (2.5\%, in $5 / 11$ rats) concentration before acquiring three additional fMRI runs.

We followed the same analysis procedure as in primates. Fig. 6A shows an example fMRI run with an asymmetric PC (a counterexample can be found in Fig. S1D). We found multiple asymmetric PCs across all fMRI runs (Fig. 6B). To select the ones most resembling burst-suppression, we focused on 
A

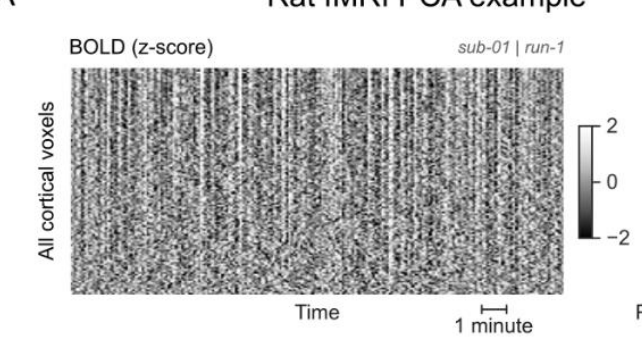

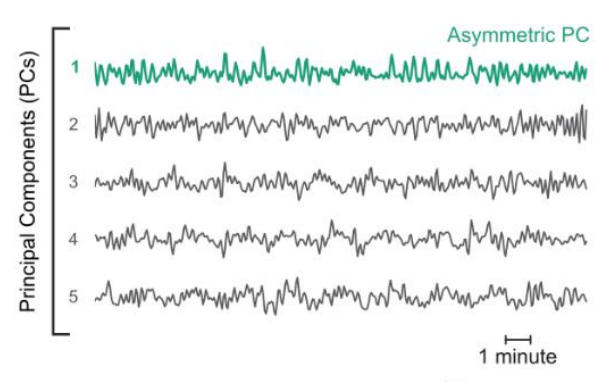
$\underset{\text { Shown slices }}{\longrightarrow}$

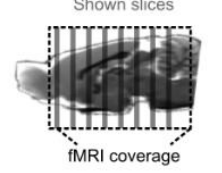

C Right

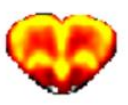

D

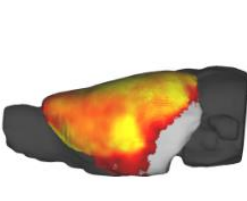

Left
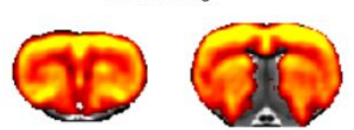

$T$ statistic (TFCE $P<0.05$ )

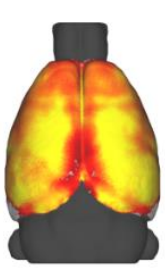

Top
Right

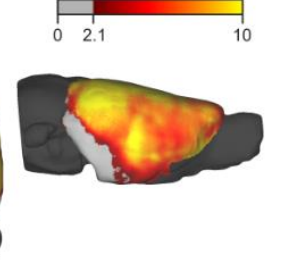

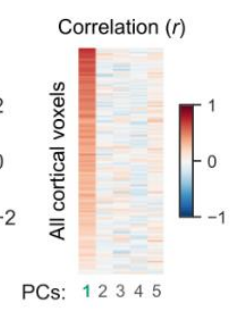

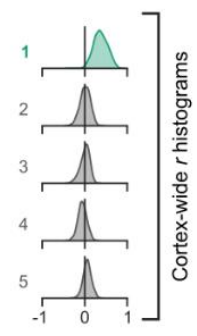

B

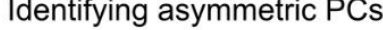

-Asymmetric PC $\left(r>0.2^{*}\right) \quad$ OOther PCs * Excluded runs
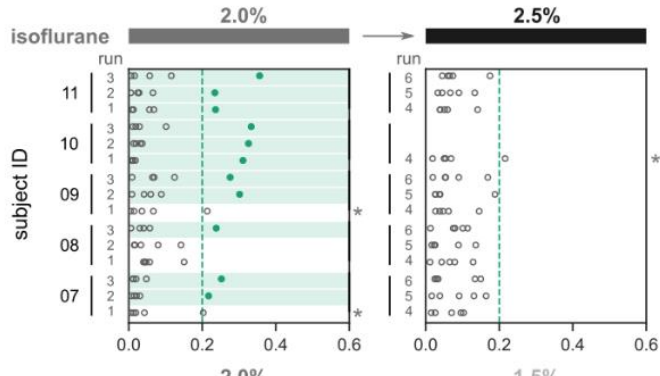

isoflurane

$$
\text { 음 }
$$
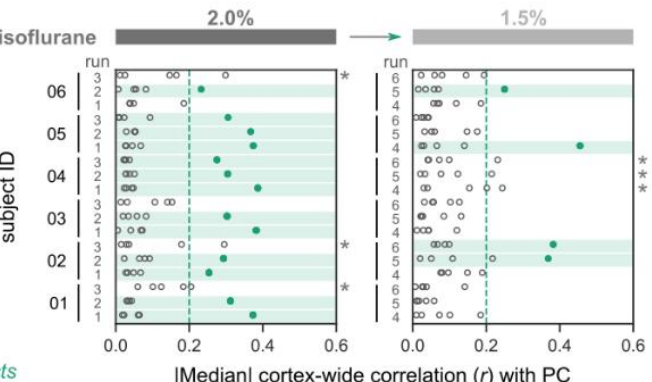

Asymmetric PC map (group)
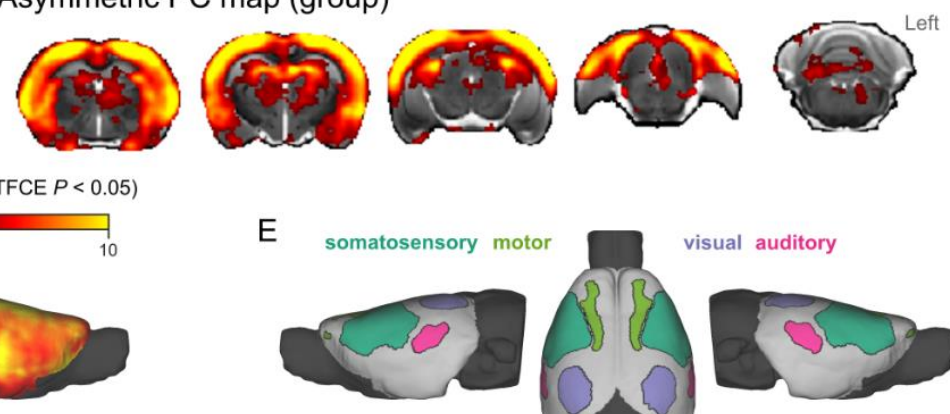

Left

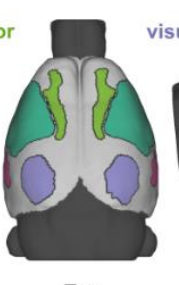

Top visual auditory

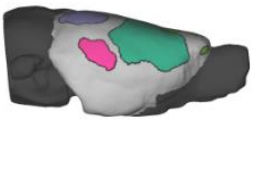

Right

Figure 6. Rats exhibit pancortical fMRI signatures of burst-suppression. (A) The cortical BOLD fMRI signal of a rat during isoflurane anesthesia is represented as a carpet plot, with rows (voxels) ordered according to their correlation with the mean cortical signal. The first five temporal principal components (PCs) of the signal are plotted below. The Pearson's correlation coefficients $(r)$ between the PCs and all cortical voxels are represented both as a heatmap and as histograms for each PC. The first PC captures the widespread fluctuation visible on the carpet plot and has an asymmetric $r$ histogram. (B) Cortex-wide median $r$ values for the first five PCs are plotted as dots across the entire rat dataset. fMRI runs with a single prominent asymmetric PC $(r>0.2$, highlighted in green) are selected, excluding runs that had a second PC within $r=0.15$ of the most asymmetric PC (marked with an asterisk). The selected 28 runs serve as inputs for general linear model (GLM) analysis. (C) The group asymmetric PC map, computed via a second-level analysis of single-subject GLMs, is shown here overlaid on a study-specific volumetric template. The group statistics were carried out with FSL randomise; the resulting $T$ statistic maps were thresholded using Threshold-Free Cluster Enhancement (TFCE) and a corrected $P<0.05$. (D) The same group map is shown on a cortical surface representation of the template. The cerebellum and the olfactory bulb are shown in dark grey. (E) The locations of primary motor, somatosensory, auditory, and visual cortices-based on the SIGMA rat brain atlas-are indicated on the surface. See also Fig. S1D, Fig. S4D, and Fig. S3. 
the subset of runs containing at least one asymmetric PC with median cortex-wide $r>0.2(37 / 64)$ but excluded nine runs that also had a second PC with relatively high median $r$ value (within $r=0.15$ of the most asymmetric PC). We were left with 28 runs, 24 of which were acquired at $2 \%$ isoflurane, and four at $1.5 \%$. We used the 28 asymmetric PCs as regressors in a GLM and constructed the group-level statistical map. The map (Fig. 6C-E) showed significant correlation with the asymmetric PCs in the striatum and the entire neocortex. Contrary to primates, the map included all primary sensory and motor cortices. The cerebellar cortex and the amygdala did not correlate with burst-suppression, while significant correlations were found in parts of the thalamus (medial) and the hippocampus (Fig. 7B). No significant anti-correlations were found.

A. Marmoset
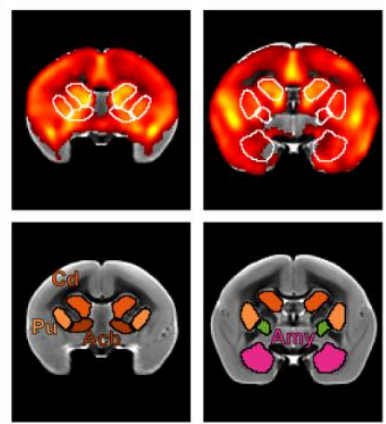

B. Rat

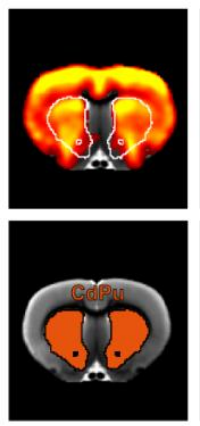

Right
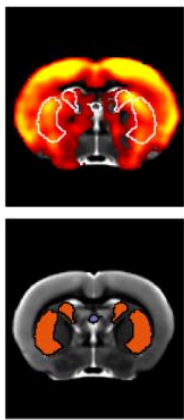
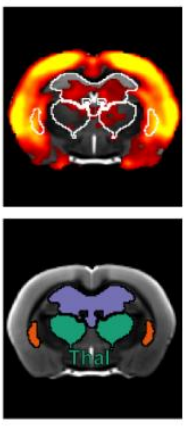

Rostral

Asymmetric PC map: group $T$ statistic (TFCE $P<0.05$ )
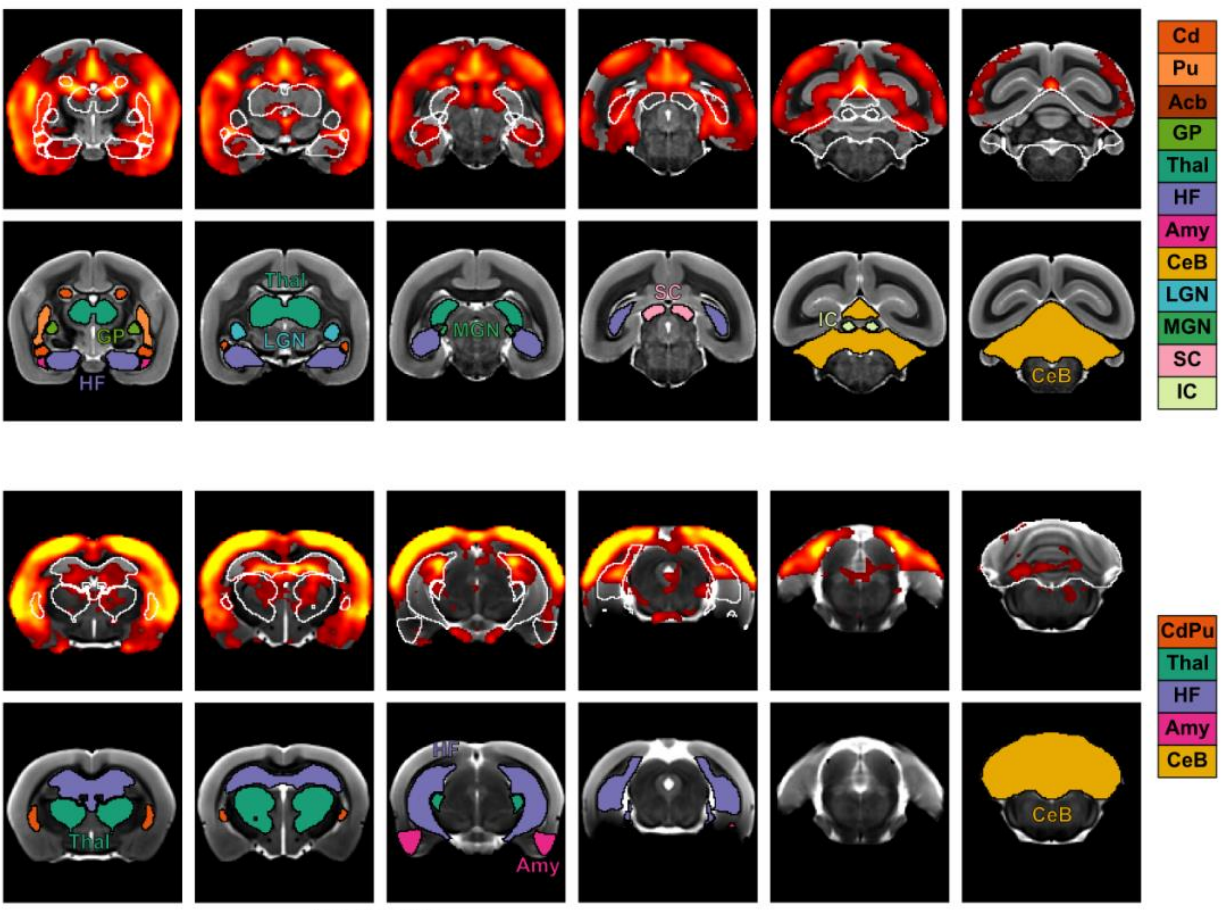

Figure 7. Subcortical structures during burst-suppression in marmosets and rats. (A) Group statistic map showing the spatial distribution of asymmetric PCs in marmosets (same as in Fig. 5C). The views are adjusted to focus on subcortical structures, on coronal slices. (B) The corresponding map is shown for rats (same as in Fig. 6C). For each species, the bottom row shows the location of major subcortical structures, defined based on the Marmoset Brain Mapping coarse subcortical atlas and the SIGMA rat brain atlas. Cd: caudate; Pu: putamen; Acb: accumbens; GP: globus pallidus; Thal: thalamus; HF: hippocampal formation; Amy: amygdala; CeB: cerebellum; LGN: lateral geniculate nucleus; MGN: medial geniculate nucleus; SC: superior colliculus; IC: inferior colliculus.

The burst-suppression map shown in Fig. 6 resembles a unified cortico-striatal network often found by BOLD fMRI studies in isoflurane-anesthetized rats (Kalthoff et al., 2013; Liu et al., 2013, 2011; Paasonen et al., 2018; Williams et al., 2010), suggesting that previous studies were potentially conducted during burst-suppression. To reinforce this notion, we revisited the data (six rats, 
anesthetized with 1.3\% isoflurane) from one study (Paasonen et al., 2018) and subjected it to our preprocessing and analysis pipeline. As shown in Fig. S3, we found a single asymmetric PC (median cortex-wide $r>0.2$ ) in all rats, with spatial distribution that matched both the unified network described in the original study (Paasonen et al., 2018) and the rat burst-suppression map in the present manuscript. The results strongly suggest that the apparent unification of cortex and striatum into a single functional network is a direct effect of burst-suppression.

\section{Primate V1 is uncoupled from the rest of the cortex during burst-suppression}

Our results so far revealed a striking difference between primates and rodents: burst-suppression in rats engaged the entire neocortex, while in the three primate species it appeared to largely spare sensory cortices. The most prominent and unambiguous example is V1, which in primates showed no correlation to the asymmetric PCs. This finding cannot be attributed to a fluke of thresholding (see unthresholded $T$ statistic maps in Fig. S4), or to local signal drop-outs (see temporal signal-to-noise ratio maps in Fig. S5).

The above prompted us to look more closely at V1 activity and to compare it with an area strongly engaged in burst-suppression. In each of the four species we defined two regions-of-interest-one in $\mathrm{V} 1$ and another in the cingulate cortex (Brodmann area 24 in primates, primary cingulate cortex in rats) - and extracted their mean BOLD signal time series from fMRI runs previously identified as burst-suppression. We then computed the standard deviation (SD) of the time series, as a measure of BOLD signal amplitude, and its power spectral density (PSD). We also performed seed-based correlation analysis using the extracted time series as GLM regressors. The results are shown in Fig. 8 across species. As expected, the cingulate exhibited the same bistable fluctuation as the asymmetric PCs and its seed-based correlation map was identical to the species-specific asymmetric PC maps. V1 activity in rats was indistinguishable from that of the cingulate across all metrics: BOLD signal amplitude $(P=0.63$, paired samples two-sided Wilcoxon rank-sum test), PSD, and seed-based correlation map. Conversely, V1 activity in primates was very different from the cingulate: BOLD signal amplitude was reduced $(P<0.001$ in humans, $P=0.004$ in macaques, $P=0.008$ in marmosets; paired samples two-sided Wilcoxon rank-sum test), PSD was lower across all frequencies, and seedbased correlation maps were confined to the V1 itself, barely extending beyond the seed borders.

We can conceive three possible mechanisms for the apparent uncoupling of V1 in primates: V1 is engaged in continuous slow waves without suppressions, it is in constant suppression, or there is a local change in neurovascular coupling. Lacking invasive neural recordings, we cannot dissect these possibilities with certainty. Nevertheless, we tried to get a hint from the human data, by examining whether V1 activity differs between the EEG-defined states of burst-suppression and continuous slow waves. We focused on 12 human subjects, in which the EEG showed clear burst-suppression at the 
bioRxiv preprint doi: https://doi.org/10.1101/2021.10.15.464515; this version posted October 16, 2021. The copyright holder for this preprint (which was not certified by peer review) is the author/funder, who has granted bioRxiv a license to display the preprint in perpetuity. It is made available under aCC-BY 4.0 International license.

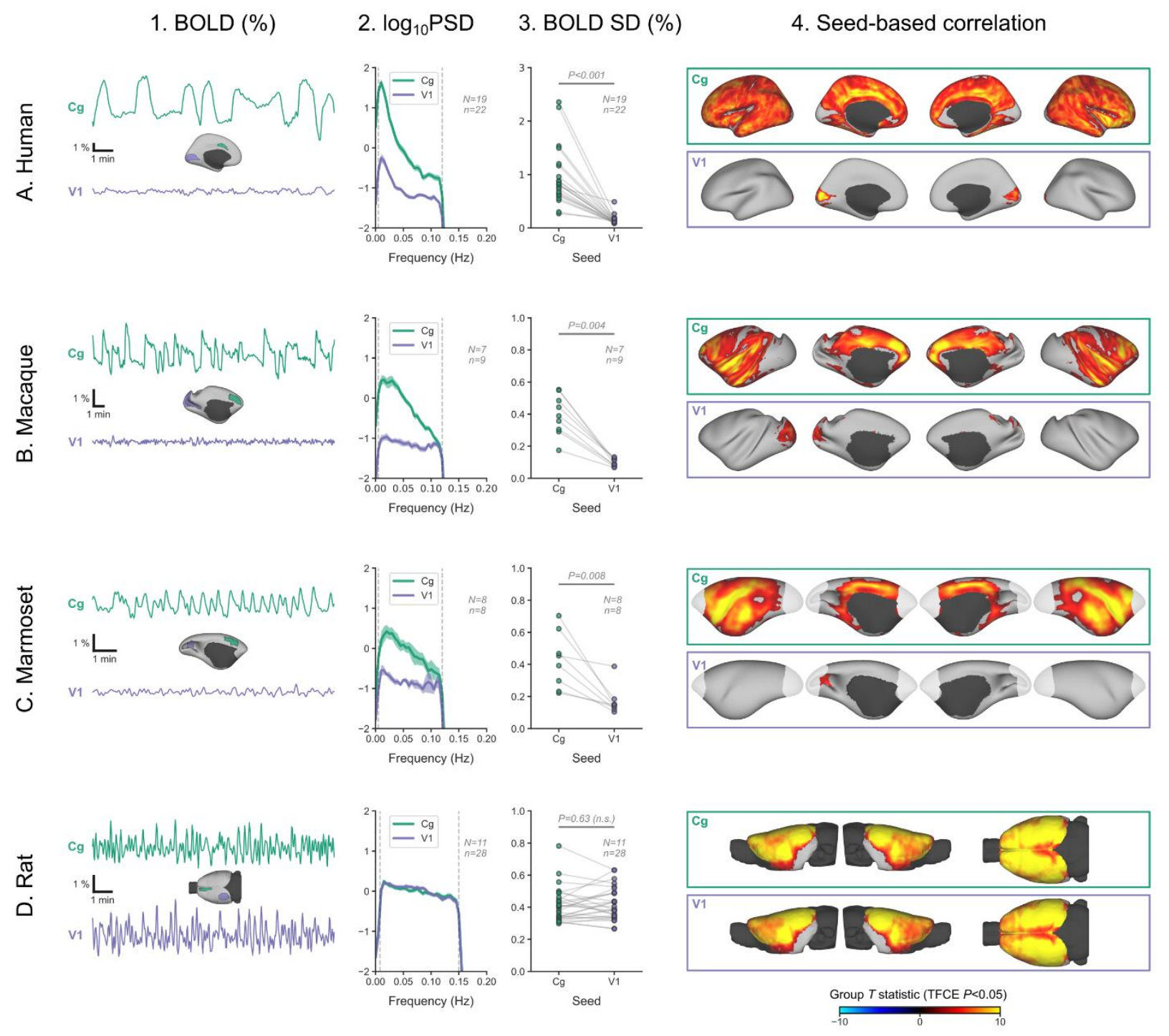

Figure 8. Primate V1 is uncoupled from the rest of the cortex during burst-suppression. (A) BOLD signal time series extracted from two regions-of-interest, the cingulate $(\mathrm{Cg})$ area $24 \mathrm{~cd}$ and the primary visual cortex (V1), are shown for an example human subject during burst-suppression (A1). The power spectral density (PSD) of the two regions' time series is plotted as mean \pm s.e.m. across all fMRI runs with burst-suppression (A2). The SD of the time series is plotted for the same fMRI runs, as a measure of BOLD signal amplitude (A3). The SDs of the two regions are compared using a paired samples two-tailed Wilcoxon rank-sum test ( $P$ values given). Panel A4 shows the results of seed-based correlation analysis-performed for each of the two regionsoverlaid on the cortical surface. The group statistics were carried out with FSL randomise; the resulting $T$ statistic maps were thresholded using Threshold-Free Cluster Enhancement (TFCE) and a corrected $P<0.05$. Panels B, C, and $\mathbf{D}$ show the exact same plots as in $\mathbf{A}$, but for macaques, marmosets, and rats, respectively. The cingulate $(\mathrm{Cg})$ seed corresponds to the macaque area $24 \mathrm{c}$, the marmoset area 24 , and the rat primary cingulate cortex. Marmoset brain areas not covered by the fMRI imaging volume are overlaid with white transparency. The fMRI runs included in the analysis are the ones with an asymmetric PC, same as in previous figures. N: number of subjects; n: number of fMRI runs. 
high sevoflurane concentration (3.9-4.6\%), and continuous slow waves at both the intermediate (3\%) and the low (2\%) concentrations. For each of V1 and cingulate seeds, we examined the effects of sevoflurane concentration on BOLD signal amplitude and PSD. The detailed results are presented in Fig. S6 and Tables S1-S2. In summary, both V1 and cingulate exhibited a reduction in BOLD signal amplitude from low to intermediate concentrations. During the high concentration (burstsuppression), the signal amplitude rose significantly in cingulate, but stayed the same as during the intermediate concentration in V1 - albeit with a slight shift towards lower frequencies. Assuming the presence of continuous slow waves in V1 during the intermediate concentration, they are likely sustained through the high concentration as well. In case of a complete suppression of slow wave activity, a noticeable further reduction in BOLD signal amplitude would be expected. A local change in neurovascular coupling, though unlikely, cannot be ruled out with the current data.

\section{Discussion}

In this study we identified the fMRI signatures of anesthesia-induced burst-suppression (Fig. 1) and constructed comparable maps in four mammalian species: humans (Fig. 2), macaques (Fig. 3), marmosets (Fig. 5), and rats (Fig. 6). The maps exhibited homology across the three primate species: burst-suppression engaged the striatum and a large part of the neocortex, with some exceptions mostly at unimodal primary areas. The primary visual area (V1) was the predominant exception, appearing entirely decoupled from the rest of the cortex (Fig. 8). Contrary to primates, burstsuppression was pancortical in rats, including all sensory and motor areas. In the following discussion, we first consider the limitations and potential applications of our method for identifying the fMRI signatures of burst-suppression, and then explore the implications of inter-species differences for existing models of burst-suppression and for future translational efforts.

\section{fMRI signatures of burst-suppression}

We determined that EEG-defined burst-suppression in humans manifests on fMRI as an 'asymmetric PC', which can be used as this state's fMRI signature (Fig. 1). The asymmetry refers to the distribution of correlation coefficients between the PC and cortical voxels and reflects profound cortical synchrony. The threshold for PC asymmetry (median cortex-wide $r$ ) is arbitrarily defined, which constitutes a weakness, since the appropriate value is expected to vary depending on animal species, anesthetic agent, and imaging parameters. To create the animal burst-suppression maps, we conservatively selected only fMRI runs that presented a single clear asymmetric PC candidate (see Figs. 3, 5, and 6). We have several reasons to be confident in our selection, despite the manually chosen thresholds. Firstly, the isoflurane concentration range at which these asymmetric PCs were detected closely matches previous reports of burst-suppression in macaques (Vincent et al., 2007; 
Zhang et al., 2019) and rats (Detsch et al., 2002; Liu et al., 2011; Masamoto et al., 2009; Stenroos et al., 2021). In this regard, our findings in rats are especially encouraging, because almost all asymmetric PCs were found at a concentration of $2 \%$, and less so at $1.5 \%$ or $2.5 \%$ (Fig. 6). Secondly, the detected widespread fluctuations cannot be attributed to known non-neural sources, such as physiology or motion. The asymmetric PCs displayed quasi-periodicity (see Fig. S7) and were overwhelmingly correlated with grey matter areas. Physiological noise related to mechanical ventilation or heartbeat would have been strictly periodic, while motion artifacts are known to distribute along image edges. In summary, all evidence points towards burst-suppression as the underlying cause of the selected asymmetric PCs in the investigated animals.

Given the arbitrary threshold limitation, our method should not be viewed as an automated fMRIbased classification of burst-suppression but instead as a heuristic algorithm for identifying this state. Nevertheless, the method can find applications within the growing animal fMRI community (Mandino et al., 2020; Milham et al., 2020) and potentially also in clinical settings. Animal fMRI researchers could use the algorithm to screen datasets for the presence of burst-suppression-as we did in Fig. S3. Nonetheless, the generalization of the method to other animal species and anesthetic agents warrants caution, keeping in mind that the amplitude and duration of bursts vary across anesthetics (Akrawi et al., 1996; Fleischmann et al., 2018). One potential clinical application concerns the management of comatose patients, where burst-suppression is often present-either transiently or throughout (Brenner, 1985; Brown et al., 2010; Cloostermans et al., 2012; Hofmeijer et al., 2014; Young, 2000). After appropriate validation, our method could map burst-suppression in these patients, even at centers lacking the capacity for simultaneous EEG-fMRI recordings. An fMRI readout of burst-suppression, with its high spatial resolution and whole-brain coverage, could provide clinicians with information inaccessible by EEG. The implications of this additional information for the management of comatose patients remain to be examined.

\section{Burst-suppression across mammalian species}

How do our maps fit in what we know about the spatial distribution of burst-suppression? The apparent exclusion of V1 replicates the previous analysis in humans (Golkowski et al., 2017) and corroborates an existing report in macaques (Zhang et al., 2019). Still, our macaque map constitutes an important generalization, as it is based on seven long-tailed macaques-compared to only two rhesus individuals in the previous study. The marmoset burst-suppression map is, to our knowledge, the first of its kind and suggests that V1 exclusion may be a general primate feature. The pancortical distribution of burst-suppression in rats agrees with all previous findings in this species, by fMRI (Aedo-Jury et al., 2020; Liu et al., 2013, 2011; Paasonen et al., 2019; Schwalm et al., 2017) or calcium imaging (Ming et al., 2021). The strength of our approach lies in computing all maps with the same data-driven approach, thus allowing us to make direct cross-species comparisons. 
The main inter-species difference was the exclusion of visual areas (and parts of other unimodal cortices) in primates, but not in rats. While bursts were traditionally viewed as synchronous cortexwide events, recent work rather describes them as complex waves (Bojak et al., 2015) emerging from a spatially shifting focus and rapidly spreading through the cortex (Ming et al., 2021). Electrocortical recordings in propofol-anesthetized human patients revealed that some electrodes could display continuous activity while others engaged in burst-suppression (Lewis et al., 2013). Together with our results, this implies that brain areas may vary in their propensity for generating and/or propagating bursts. Regional metabolic differences could underlie such variability, according to a proposed neurophysiological-metabolic model of burst-suppression (Ching et al., 2012). This model posits that suppressions occur when metabolic resources can no longer sustain neuronal firing, whereas bursts constitute transient recoveries of activity—enabled by slow metabolic replenishment. The visual cortex of primates could impose special metabolic demands and thus find itself at an extreme end of the spectrum for burst propensity. Such special demands could arise from its many distinctive characteristics-including cytoarchitecture, neurotransmitter receptor expression (Froudist-Walsh et al., 2021), functional connectivity profile (Margulies et al., 2016), and cortical myelin density (Essen et al., 2019). We particularly suspect cortical myelin to play a role, considering that many areas excluded from our primate burst-suppression maps-V1, MT, primary motor, and somatosensory cortices-are among the richest in myelin (Essen et al., 2019). Conversely, rodents exhibit a more uniform myelin distribution (Fulcher et al., 2019), which could translate in a likewise uniform propensity for bursting.

Whatever the cause, the non-correlation of V1 and other primary sensory areas with burstsuppression challenges existing ideas about the origin of bursts. Studies in isoflurane-anesthetized humans, cats, rats, and mice showed that bursts can be evoked by incoming sensory stimuli, including flashes of light (Hartikainen et al., 1995; Hudetz and Imas, 2007; Kroeger and Amzica, 2007; Land et al., 2012). Consequently, burst-suppression has been described as a state of cortical hypersensitivity, during which even subliminal sensory stimuli can trigger a burst excitation (Kroeger and Amzica, 2007). In that case, bursts would be expected to originate at the stimulated sensory area and then spread throughout the cortex indiscriminately, like ripples on a pond (Muller et al., 2018; SanchezVives et al., 2017; Schwalm et al., 2017; Stroh et al., 2013). The pancortical rodent map is fully compatible with this view, but the exclusion of sensory areas in primates seems paradoxical: why would the areas of origin not be part of the ripple? A possible explanation is that the bursts captured in our data were internally generated or evoked through a non-excluded sensory modality (e.g., auditory areas in nonhuman primates). Nevertheless, a closer investigation of sensory-evoked bursts in primates is warranted-especially with visual stimuli. 
So far, we have discussed cortical aspects of burst-suppression, but fMRI also offers access to subcortical structures (see Figs. 4 and 7). Among these, the striatum displayed the strongest correlation with burst-suppression across all four species-showing that the cortex does not act in isolation. Cortico-striatal loops may play an important-but yet unexplored-role in burst propagation. The thalamus, on the contrary, was only partly correlated with burst-suppression, and to a varying extent across species. Interestingly, anteromedial parts were more likely to be involved than posterolateral parts, possibly reflecting a differential role played by thalamic primary relay centers and higher-order nuclei, as previously hypothesized (Ming et al., 2021). Regional variations in thalamic activity could even shape cortical differences, since the thalamus may be important for driving burst onsets (Ming et al., 2021; Steriade et al., 1994). Similar to the thalamus, the hippocampus and amygdala were partially correlated with burst-suppression. However, we refrain from concrete conclusions, since in primates these two structures are located in low signal-to-noise areas and often suffer from susceptibility-induced distortions (see Fig. S5). The cerebellum also suffered from low signal-to-noise in our nonhuman primate data and was only partly captured in marmosets and rats. Nevertheless, the non-correlation of the cerebellar cortex with burst-suppression is likely a true effect, given its consistency across all species.

Before closing, we wish to address a potentially confusing issue of terminology. We employ the term 'burst-suppression', preferred by clinical anesthesiologists. It has been pointed out (Ming et al., 2021) that several rodent studies may refer to the same phenomenon as 'slow oscillation' (Aedo-Jury et al., 2020; Sanchez-Vives et al., 2017; Schwalm et al., 2017; Stroh et al., 2013)—meaning a $<1 \mathrm{~Hz}$ alternation between a depolarized 'UP' state of neural activity and a hyperpolarized 'DOWN' state of silence (Steriade et al., 1993). In the 'slow oscillation' context, suppressions can be viewed as prolonged DOWN states (Ching et al., 2012; Kroeger and Amzica, 2007). However, the line between the two is blurry, given the gradual dose-dependent lengthening of quiescent periods. This distinction is even harder if one considers potential inter-species differences in the timescale of burstsuppression. We noticed that bursts and suppressions alternate faster when moving from humans to nonhuman primates to rats (Fig. S7). Within the framework of the neurophysiological-metabolic model of burst-suppression (Ching et al., 2012), such differences in timescale could reflect the faster metabolic rate of smaller animals. Verifying this hypothesis would require further studies involving direct neural recordings.

Summing up, our findings in primates oppose the view of bursts as global cortical events and imply a varying propensity of brain regions to generate and/or propagate bursts. By contrast, burstsuppression in rats appears to be pancortical-prompting an investigation into the cause of this primate-rodent difference and raising questions about the validity of rodent models for anesthesia and coma. Addressing these issues will necessitate invasive neural recordings during burst- 
suppression, in both primates and rodents, with V1 being of highest interest. Our findings also point towards several other promising research directions: clarifying the exact relationship between spontaneous and sensory-evoked bursts, investigating the role of the striatum and thalamus, and examining inter-species differences in the timescale of burst-suppression.

\section{Acknowledgements}

This work was supported by the DFG Center for Molecular Physiology of the Brain (DFG project number 5485646) and the Leibniz Science Campus Primate Cognition. We wish to thank: Kristin Kötz and Kerstin Fuhrmann for technical assistance; Dr. Iris Steinmann for advice concerning EEG data analysis; Dr. Myrto Panopoulou, Tor Rasmus Memhave, and Christina Kajba for proof-reading.

\section{Author Contributions}

Conceptualization, N.S. and S.B.; methodology, N.S. and S.B.; software, N.S.; validation: N.S., M.O.-R., J.B., and S.B.; formal analysis, N.S.; investigation, N.S., J.M., and S.B.; resources, J.P., O.G., D.G., A.R., and R.I.; data curation, N.S., J.P., and D.G.; writing - original draft, N.S.; writing - review \& editing, all authors; visualization, N.S.; supervision, S.B.; project administration: N.S. and S.B., funding acquisition, S.B., O.G., and R.I.

\section{Competing Interests}

The authors declare no competing interests.

\section{Data and Code Availability}

The custom python code implementing the carpet plots and PCA analysis is publicly available through GitHub (https://github.com/niksirbi/pcarpet/) and Zenodo (doi: 10.5281/zenodo.5545696). All animal MRI datasets will be made open access by the time of publication in a scientific journal. 


\section{Materials and Methods}

\section{Experimental subjects and anesthesia}

Five datasets from three research sites were used for this manuscript (see Table 1). The human data were obtained from a previous study that have been described in three other articles (Golkowski et al., 2019, 2017; Ranft et al., 2016). Experimental details for the second rat dataset (Rat 2) have also been presented elsewhere (Paasonen et al., 2018, 2016). All other experiments were carried out at the German Primate Center (Deutsches Primatenzentrum GmbH, Göttingen, Germany) with the approval of the ethics committee of the Lower Saxony State Office for Consumer Protection and Food Safety and in accordance with the guidelines from Directive 2010/63/EU of the European Parliament on the protection of animals used for scientific purposes. The relevant approval numbers are: 33.1942502-04-16/2278 (Macaque), 33.19-42502-04-17/2496 (Marmoset), 33.19-42502-04-15/2042 (Rat 1). All animals were purpose-bred, raised, and kept in accordance with the German Animal Welfare Act and the high institutional standards of the German Primate Center.

Table 1. Datasets of the present study. For age and weight, mean and range values are reported. Anesthetic concentration refers to the range used during fMRI acquisition. In humans and macaques the measured endtidal concentration of the anesthetic gas is reported, in marmosets and rats the output concentration of the vaporizer.

\begin{tabular}{llllll}
\hline & Human & Macaque & Marmoset & Rat 1 & Rat 2 \\
\hline Species/Strain & - & M. fascicularis & C. jacchus & Wistar & Wistar \\
Site & Munich & Göttingen & Göttingen & Göttingen & Kuopio \\
Field strength & $3 \mathrm{~T}$ & $3 \mathrm{~T}$ & $9.4 \mathrm{~T}$ & $9.4 \mathrm{~T}$ & $7 \mathrm{~T}$ \\
Subjects (N) & 20 & 13 & 20 & 11 & 6 \\
Sex & $\mathrm{M}$ & $\mathrm{F}$ & $10 \mathrm{~F}$ & $\mathrm{~F}$ & $\mathrm{M}$ \\
Age (years) & $26(20-36)$ & $13.7(6.8-19.8)$ & $6.1(1.9-14.2)$ & - & - \\
Body Weight & - & $5.4 \mathrm{~kg}(3.6-8.1)$ & $407 \mathrm{~g}(337-517)$ & $398 \mathrm{~g}(350-450)$ & $307 \mathrm{~g}(265-350)$ \\
Anesthetic & Sevoflurane & Isoflurane & Isoflurane & Isoflurane & Isoflurane \\
Concentration (\%) & $2.0-4.6$ & $0.95-1.50$ & $0.6-1.1$ & $1.5-2.5$ & 1.3 \\
\hline
\end{tabular}

Human. The human EEG-fMRI data were acquired in 20 healthy adult men aged 20-36 years (mean 26). Anesthesia was induced by delivering a gradually increasing concentration of sevoflurane through a tight-fitting face mask. When clinically indicated, a laryngeal mask was inserted, and mechanical ventilation was started. Following that, sevoflurane concentration was increased until the 
EEG showed suppression about $50 \%$ of the time, with suppression periods lasting at least $1 \mathrm{~s}$ (reached at end-tidal concentrations of 3.9-4.6\%). At this point, $700 \mathrm{~s}$ of simultaneous EEG-fMRI were recorded. This recording was repeated at two lower fixed concentrations, $3 \%$ and $2 \%$, each following an equilibration period of $15 \mathrm{~min}$. The lowest concentration recording was only acquired in 15/20 participants.

Macaque. The macaque study included 15 female long-tailed macaques (Macaca fascicularis) in total, but fMRI data of sufficient duration (minimum $10 \mathrm{~min}$ ) and quality were obtained only in 13/15. The macaques varied widely in age (6.8-19.8 years, mean 13.7) and weight (3.6-8.1 kg, mean 5.4). Prior to the imaging session, the animals were given no food for six hours and no water for two hours. Anesthesia was induced with an intramuscular (i.m.) injection of ketamine (3-10 mg/kg, Ketamin $10 \%$, Medistar) and medetomidine (0.015-0.03 mg/kg, Dorbene vet $1 \mathrm{mg} / \mathrm{ml}$, Zoetis). The animals were intubated using an endotracheal tube and placed in a sphinx position inside a custom-made MRI-compatible stereotaxic frame to minimize movement artifacts. The ear bars also served as hearing protection and were dabbed with a lidocaine-containing ointment (EMLA 5\%, AstraZeneca) for local anesthesia. Dexpanthenol eye ointment (Bepanthen, Bayer) was applied for corneal protection. Body temperature was maintained constant at $37^{\circ} \mathrm{C}$ with the help of a circulating warm water system. Monitoring equipment was attached, including a pulse oximeter (NONIN model 7500FO, SANIMED GmbH, Germany), a respiratory belt, surface electrodes for electrocardiogram (MRI ECG electrodes, CONMED, USA), and an anesthetic gas analyzer (Philips M1026A Anesthesia Gas Module) for measuring $\mathrm{CO}_{2}$ and isoflurane concentrations. Throughout the imaging session, the animals were mechanically ventilated with a respirator (Siemens-Elema AB SV 900C Servo Ventilator) at a rate of 8-14 bpm, and anesthesia was maintained with isoflurane, with the concentration being continually adjusted to ensure physiological stability. The goal was to sustain an anesthetized depth that would ensure endotracheal tube toleration. The needed end-tidal isoflurane concentrations varied between $0.9-2 \%$ across animals. Each imaging session lasted for up to five hours, during which multiple structural MRI contrasts were acquired, as well as task-free BOLD fMRI (one or two runs per animal, each lasting 600-1200 s). During fMRI acquisition, end-tidal isoflurane concentrations ranged $0.95-1.5 \%$ across animals. At the end of the imaging session isoflurane was stopped and the macaques were extubated as soon as spontaneous breathing was safely established.

Marmoset. The marmoset study included a total of 35 common marmosets (Callithrix jacchus) of both sexes, but BOLD fMRI data was only obtained in 20 of those (10 females). The animals varied widely in age (1.9-14.2 years, mean 6.1) and weight (337-517 g, mean 407). Anesthesia was induced with a mixture of alfaxalone $(12 \mathrm{mg} / \mathrm{kg}$ ) and diazepam ( $3 \mathrm{mg} / \mathrm{kg}$ ) injected i.m. This was followed by $0.05 \mathrm{ml}$ glycopyrronium bromide per animal (Robinul $0.2 \mathrm{mg} / \mathrm{ml}$, Riemser Biosyn) to prevent secretions, maropitant (1 mg/kg, Cerenia, Pfizer) as an antiemetic, and meloxicam (0.2 mg/kg, Metacam, 
Boehringer Ingelheim) as an anti-inflammatory analgesic. The animals were intubated using a custom-made flexible endotracheal tube and mechanically ventilated at a rate of 35-37 bpm (Animal Respirator Advanced 4601-2; TSE Systems GmbH, Bad Homburg, Germany). The marmosets were placed in a prone position inside a custom-built MRI-compatible bed equipped with a bite bar and ear bars. The ear bars also served as hearing protection and were dabbed with a lidocaine-containing ointment (EMLA 5\%, AstraZeneca) for local anesthesia. Dexpanthenol eye ointment (Bepanthen, Bayer) was applied for corneal protection. Monitoring equipment consisted of a rectal temperature probe, a pneumatic pressure sensor placed on the chest, and three surface electrodes for ECG (MRcompatible Model 1030 monitoring and gating system; Small Animal Instruments Inc., Stony Brook, NY 11790, USA). Rectal temperature was kept within $36.5 \pm 1{ }^{\circ} \mathrm{C}$ using a pad heated by circulating water. Anesthesia was maintained with isoflurane delivered via the respirator, using a mixture of medical air $/ \mathrm{O}_{2}$ (1:1 ratio) as the carrier gas. The isoflurane concentration was adjusted individually for each animal, ranging $0.6-1.1 \%$, in order to maintain stable anesthesia and physiology. Each imaging session lasted for up to five hours, during which multiple structural MRI contrasts were acquired, as well as one task-free fMRI run lasting $600 \mathrm{~s}$. At the end of the session isoflurane was stopped, and the marmosets were extubated as soon as spontaneous breathing was safely established.

Rat 1. The Rat 1 dataset included 11 female adult Wistar rats (Charles Rivers Laboratories, Sulzfeld, Germany), weighing 350-450 g (mean 398). The rats were group-housed in cages with environmental enrichment, at a $12 / 12$-hour light/dark cycle, with $20-24{ }^{\circ} \mathrm{C}$ temperature and $45-55 \%$ humidity. Water and standard chow were provided ad libitum. Anesthesia was induced in a chamber with $5 \%$ isoflurane in $100 \% \mathrm{O}_{2}$. After the loss of the righting reflex, isoflurane was reduced to $2-3 \%$ and delivered through a nose cone. The rats were then intubated using a custom-made flexible tracheal tube and mechanically ventilated at a rate of $30 \mathrm{bpm}$ (Animal Respirator Advanced 4601-2; TSE Systems $\mathrm{GmbH}$, Bad Homburg, Germany). Isoflurane was stabilized at 2\%, using medical air $/ \mathrm{O}_{2}$ mixture (1:1 ratio) as the carrier gas. The animals were fixed inside a custom-built MRI-compatible rat holder in a supine position (Sirmpilatze et al., 2019) and dexpanthenol eye ointment (Bepanthen, Bayer) was applied for corneal protection. Monitoring equipment was attached, consisting of a rectal temperature probe, a pneumatic pressure sensor placed on the chest, and three subcutaneous needle electrodes for ECG (MR-compatible Model 1030 monitoring and gating system; Small Animal Instruments Inc., Stony Brook, NY 11790, USA). Rectal temperature was kept within $36.5 \pm 1^{\circ} \mathrm{C}$ using a pad heated by circulating water. The rat was placed at the isocenter of the MRI system and structural MRI data were acquired. These were followed by three task-free fMRI runs, each $360 \mathrm{~s}$ long. After that, isoflurane was either decreased to $1.5 \%$ (6/11 animals) or increased to 2.5\% (5/11 animals). Following an equilibration period of $30 \mathrm{~min}$, three additional task-free fMRI runs were acquired (six runs per animal in total). In one rat only four fMRI runs could be obtained, due to an early interruption 
of the imaging session (disconnection of the endotracheal tube). At the end of the imaging session isoflurane was stopped and the rats were extubated as soon as spontaneous breathing had recovered.

Rat 2. The six adult male Wistar rats included in the Rat 2 dataset (Paasonen et al., 2018, 2016) weighed 265-350 g (mean $307 \mathrm{~g}$ ). Briefly, anesthesia was induced with isoflurane 5\%, maintained at $2 \%$ during animal preparation, and at $1.3 \%$ during imaging. The rats were endotracheally intubated and mechanically ventilated, with $\mathrm{N}_{2}: \mathrm{O}_{2}$ 70:30 mixture being used as the carrier gas. The data analyzed for this study comprised a single 300 s run of task-free fMRI per animal.

\section{MRI acquisition}

Human. Human data were acquired on a $3 \mathrm{~T}$ whole-body MRI system (Achieva Quasar Dual 3.0T $16 \mathrm{CH}$, Amsterdam, Netherlands) with an 8-channel phased-array head coil. fMRI data were collected using a gradient-echo echo planar imaging sequence (EPI) sequence with the following parameters: repetition time $2 \mathrm{~s}$, echo time $30 \mathrm{~ms}$, flip angle $75^{\circ}$, field of view $220 \times 220 \mathrm{~mm}^{2}$, matrix size 96 × 96 (in-plane resolution of $2.3 \times 2.3 \mathrm{~mm}^{2}$ ), 35 axial slices with $3 \mathrm{~mm}$ thickness and $1 \mathrm{~mm}$ interslice gap, acquisition time $700 \mathrm{~s}$ (350 volumes). A $1 \mathrm{~mm}$ isotropic T1-weighted image was acquired before the fMRI runs to serve as each subject's anatomical reference.

Macaque. Macaque data were acquired on a 3 Tesla whole-body MRI system (Siemens MAGNETOM Prisma 3T, Siemens Healthcare $\mathrm{GmbH}$, Erlangen, Germany). The body coil was used for signal transmission and the $70 \mathrm{~mm}$ loop coil for reception. fMRI data were collected using a gradient-echo EPI sequence with the following parameters: repetition time $2 \mathrm{~s}$, echo time $27 \mathrm{~ms}$, flip angle $90^{\circ}$, field of view $96 \times 96 \mathrm{~mm}^{2}$, matrix size $80 \times 80$ (in-plane resolution of $1.2 \times 1.2 \mathrm{~mm}^{2}$ ), 33 contiguous axial slices with $1.2 \mathrm{~mm}$ thickness, acquisition time 600-1200 s (300-600 volumes). For anatomical reference, a $0.5 \mathrm{~mm}$ isotropic T1-weighted image was collected using the MPRAGE sequence with the following parameters: 2 averages, repetition time $2.7 \mathrm{~s}$, inversion time $850 \mathrm{~ms}$, echo time $2.76 \mathrm{~ms}$, flip angle $8^{\circ}$, field of view $12.8 \times 10.8 \mathrm{~cm}^{2}$, matrix size 256 × 216, 192 contiguous axial slices with $0.5 \mathrm{~mm}$ thickness.

Marmoset. Marmoset data were acquired on a 9.4 T Bruker BioSpec MRI system, equipped with the B-GA 20S gradient, and operated via ParaVision 6.0.1 software (Bruker BioSpin MRI GmbH, Ettlingen, Germany). Signal was transmitted with a volume resonator (inner diameter $154 \mathrm{~mm}$, Bruker BioSpin MRI GmbH) and received with a 40 x $43 \mathrm{~mm}$ loop coil (Rapid Biomedical GmbH, Rimpar, Germany). A field map was measured and shims were adjusted to ensure homogeneity in an ellipsoidal volume within the marmoset brain (MAPSHIM). fMRI data were collected using a gradient-echo EPI sequence with the following parameters: repetition time $2 \mathrm{~s}$, echo time $18 \mathrm{~ms}$, flip angle $90^{\circ}$, field of view 62.4 x $25.6 \mathrm{~mm}^{2}$, matrix size 156 x 64 (in-plane resolution of $0.4 \times 0.4 \mathrm{~mm}^{2}$ ), 30 contiguous coronal slices 
with $0.8 \mathrm{~mm}$ thickness, acquisition time $600 \mathrm{~s}$ (300 volumes). The slices did not cover the entire brain in the rostro-caudal direction: they extended approximately from the anterior end of the corpus callosum to the middle of the cerebellum (see fMRI coverage in Fig. 5). For anatomical reference, we used a $0.21 \mathrm{~mm}$ isotropic magnetization transfer (MT)-weighted image, acquired using a 3D, RFspoiled, fast low angle shot (FLASH) sequence with the following parameters: 2 averages, repetition time $16.1 \mathrm{~ms}$, echo time $3.8 \mathrm{~ms}$, flip angle $5^{\circ}$, field of view 37.8 × 37.8 x $37.8 \mathrm{~mm}^{3}$, matrix size $180 \mathrm{x}$ $180 \times 180$.

Rat 1. Rat data were acquired on a 9.4 T Bruker BioSpec MRI system, equipped with the B-GA $12 \mathrm{~S} 2$ gradient, and operated via ParaVision 6.0.1 software (Bruker BioSpin MRI GmbH, Ettlingen, Germany). Signal was transmitted with a volume resonator (inner diameter $86 \mathrm{~mm}$ ) and received with a rat brain 4-channel quadrature surface coil (both from Bruker BioSpin). A field map was measured and shims were adjusted to ensure homogeneity in an ellipsoidal volume within the rat brain (MAPSHIM). fMRI data were collected using a gradient-echo EPI sequence with the following parameters: repetition time $2 \mathrm{~s}$, echo time $15 \mathrm{~ms}$, flip angle $70^{\circ}$, field of view $25.6 \times 19.2 \mathrm{~mm}^{2}$, matrix size 128 x 96 (in-plane resolution of $0.2 \times 0.2 \mathrm{~mm}^{2}$ ), 40 contiguous coronal slices with $0.5 \mathrm{~mm}$ thickness, acquisition time 720 s (360 volumes). The fMRI slices covered the entire rat brain, excluding the olfactory bulbs and the caudal $1 / 4$ of the cerebellum (see fMRI coverage in Fig. 6). For anatomical reference, a T2-weighted image was acquired using the TurboRARE (rapid acquisition with relaxation enhancement) sequence with the following parameters: 2 averages, RARE factor 8, repetition time $6.3 \mathrm{~s}$, effective echo time $40 \mathrm{~ms}$, flip angle $90^{\circ}$, field of view $32 \times 32 \mathrm{~mm}^{2}$, matrix size 256 × 256 (in-plane resolution $0.125 \times 0.125 \mathrm{~mm}^{2}$ ), 50 contiguous coronal slices with $0.5 \mathrm{~mm}$ thickness.

Rat 2. Data were acquired on a 7 T Bruker PharmaScan MRI system, operated via ParaVision 5.1 software (Bruker BioSpin MRI GmbH, Ettlingen, Germany). Signal was transmitted with a volume resonator (inner diameter $72 \mathrm{~mm}$ ) and received with a rat brain 4-channel quadrature surface coil (both from Bruker BioSpin). fMRI data were collected using a spin-echo EPI sequence with the following parameters: repetition time $2 \mathrm{~s}$, echo time $45 \mathrm{~ms}$, field of view $25 \times 25 \mathrm{~mm}^{2}$, matrix size 64 x 64 (in-plane resolution of $0.39 \times 0.39 \mathrm{~mm}^{2}$ ), 9 contiguous coronal slices with $1.5 \mathrm{~mm}$ thickness, acquisition time $600 \mathrm{~s}$ (300 volumes). The fMRI slices were placed from the posterior end of the olfactory bulb to the anterior end of the cerebellum (see fMRI coverage in Fig. S3).

\section{MRI preprocessing}

To facilitate inter-species comparability, we aimed for harmonizing preprocessing across datasets. The fMRI preprocessing steps were kept the same, but their parameters were adapted to each dataset. We used functions from multiple freely available neuroimaging toolkits, including ANTs v2.1.0 
(Advanced Normalization Tools, Avants et al., 2011), FSL v5.0.1 (FMRIB Software Library, Jenkinson et al., 2012), AFNI v18.2.06 (Cox, 1996), Freesurfer v6.0.0 (Fischl, 2012), and Connectome Workbench v1.4.2 (Marcus et al., 2011). Functions from these toolkits were combined into pipelines using the Nipype (v1.2) framework (Gorgolewski et al., 2011). The exact steps and functions used will be presented in detail for each dataset. We will also describe the species-specific standard coordinate spaces (templates) and atlases (parcellations) that were used for group analysis and data visualization.

Templates and atlases. For human data, we used the MNI152 template (MNI-ICBM average of 152 T1-weighted MRI scans, nonlinear $6^{\text {th }}$ generation (Grabner et al., 2006, distributed with FSL v5.0.1) as the standard coordinate system. For visualization purposes, results were resampled from the MNI152 volumetric space to the fsaverage surface template (Fischl et al., 1999, part of Freesurfer v6.0.0) using the registration-fusion approach (RF-ANTS, Wu et al., 2018). Cortical regions-of-interest were taken from the Human Connectome Project multi-modal parcellation 1.0 (Glasser et al., 2016) and transformed to the MNI152 and fsaverage spaces. Subcortical regions were defined based on the Harvard-Oxford subcortical structural atlas (distributed with FSL v5.0.1). For marmoset data, we used the NIH v3.0 population template (Liu et al., 2021) as a standard coordinate system. The volumetric results were visualized on the template's cortical surface (Connectome Workbench volume-to-surface-mapping, ribbon-constrained method). Regions-of-interest were taken from the Marmoset Brain Mapping V1 MRI-based parcellation (Liu et al., 2018). For the macaque and rat data, we built our own study-specific volumetric templates, using a validated template creation process implemented in ANTs (Avants et al., 2010). This process relies on iterative nonlinear registration to produce an unbiased average of the population. Our workflow was constructed similarly to the NMT template of the rhesus macaque (Seidlitz et al., 2018). For each species, the anatomical images (T1weighted for macaques, T2-weighted for rats) were corrected for variations in image intensity (ANTs N4BiasFieldCorrection, Tustison et al., 2010) and rigidly aligned using ANTs (antsRegistration). The voxel-wise average of the rigidly aligned images served as the initial target for the iterative template creation process (ANTs buildtemplateparallel.sh). The resulting template images were segmented semi-manually with ITK-SNAP (Yushkevich et al., 2006) to create masks for brain and cortex. Macaque regions-of-interest were defined based on the cortical hierarchical (CHARM, Jung et al., 2021) and subcortical (SARM, Hartig et al., 2021) atlases of the rhesus macaque, which were transformed from the NMT v2 space to our macaque template space. Rat regions-of-interest were based on the SIGMA rat brain atlas (Barrière et al., 2019) and were transformed from the SIGMA space. We reconstructed the study-specific macaque template's cortical surface (white matter and pial) using the precon_all pipeline (https://github.com/neurabenn/precon all) and resampled the volumetric results to it (Connectome Workbench volume-to-surface-mapping, ribbon-constrained method). The rat brain surface was reconstructed by extracting the isosurface of the brain mask 
(Freesurfer) and smoothing it (Connectome Workbench). For representing volumetric results on the rat cortical surface, we adapted a method previously used for the mouse brain (Huntenburg et al., 2021). In short, we first computed the surface normals, i.e. vectors perpendicular to each face of the surface mesh. We then generated line segments along those normals, with each segment stretching from the inner edge of the cortical mask towards the pial surface. Finally, we averaged the cortical voxels overlapping with each of these line segments and assigned their mean value to the corresponding surface point.

Human. MRI images were converted from PAR/REC to NIfTI (Neuroimaging Informatics Technology Initiative) format using dcm2niix v1.0 (Li et al., 2016). Structural T1-weigthed images were processed using Freesurfer's recon-all command, which includes brain extraction and segmentation. The extracted brains were registered to the MNI152 template using linear transforms (antsRegistration affine, 12 degrees of freedom), followed by nonlinear symmetric diffeomorphic registration (antsRegistration SyN, Avants et al., 2008). The fMRI time series were corrected for slice-timing (FSL slicetimer) and motion (FSL MCFLIRT), and the brain was extracted (FSL BET). Polynomial trends were removed up to the $3^{\text {rd }}$ degree, the time series were bandpass filtered at $0.005-0.12 \mathrm{~Hz}$, and spatially smoothed using a 3D gaussian kernel with full-width-at-half-maximum (FWHM) of $4 \mathrm{~mm}$ (AFNI 3dTProject). A rigid transformation matrix was calculated between the mean brain-extracted fMRI image and the brain-extracted structural image of the same subject (antsRegistration). The matrix was combined with the previously calculated structural-to-template transforms into a composite warp file, which was used to resample the preprocessed fMRI time series into the template space, at $2 \mathrm{~mm}$ isotropic resolution (antsApplyTransforms).

Macaque. MRI images were converted from DICOM to NIfTI format (dcm2niix) and rotated from the sphinx into standard orientation. Structural T1-weighted images were corrected for intensity inhomogeneities (ANTs N4BiasFieldCorrection) and registered to the study-specific macaque template (antsRegistration affine, followed by SyN). The template's brain mask was transformed back to the single-subject structural space and the brain was extracted. The fMRI time series were corrected for slice-timing (FSL slicetimer) and motion (FSL MCFLIRT), and the brain was extracted semi-manually with ITK-SNAP. Polynomial trends were removed up to the $3^{\text {rd }}$ degree, the time series were bandpass filtered at $0.005-0.12 \mathrm{~Hz}$, and spatially smoothed using a 3D gaussian kernel with FWHM of $2 \mathrm{~mm}$ (AFNI 3dTProject). The mean brain-extracted fMRI image was rigidly registered to the same subject's structural brain image (antsRegistration). The resulting transformation matrix was combined with the previously calculated structural-to-template transforms into a composite warp file, which was used to resample the preprocessed fMRI time series into the template space, at $1 \mathrm{~mm}$ isotropic resolution (antsApplyTransforms). 
Marmoset. MRI images were exported from ParaVision 6.0.1 to DICOM format, converted from DICOM to NIfTI (dcm2niix), and rotated into standard orientation. Structural images (MT-weighted) were corrected for intensity inhomogeneities (ANTs N4BiasFieldCorrection) and registered to the T2weighted image of the NIH v3.0 population template (antsRegistration affine, followed by SyN). The fMRI time series were corrected for slice-timing (FSL slicetimer) and motion (antsMotionCorr). Polynomial trends were removed up to the $3^{\text {rd }}$ degree, the time series were bandpass filtered at $0.005-0.12 \mathrm{~Hz}$, and spatially smoothed using a 3D gaussian kernel with FWHM of $1 \mathrm{~mm}$ (AFNI 3dTProject). The mean fMRI image was rigidly registered to the subject's structural image (antsRegistration). The resulting transformation matrix was combined with the previously calculated structural-to-template transforms into a composite warp file, which was used to resample the preprocessed fMRI time series into the template space, at $0.4 \mathrm{~mm}$ isotropic resolution (antsApplyTransforms).

Rat. The rat preprocessing pipeline was identical to the one used for marmosets, with a few differences. The registration target for structural T2-weighted images was our study-specific rat brain template. The 3D gaussian kernel's FWHM was set to $0.6 \mathrm{~mm}$ and the preprocessed fMRI time series were resampled into the template space at a resolution of $0.2 \times 0.2 \times 0.5 \mathrm{~mm}^{3}$. Bandpass temporal filtering was initially applied using the same cutoffs as for the other species $(0.005-0.12 \mathrm{~Hz})$. However, we later realized that bursting activity in rats also occurred at faster timescales (see Fig. S7) and reanalyzed the data using higher bandpass cutoffs $(0.008-0.15 \mathrm{~Hz})$. This gave a richer representation of burst-suppression in the temporal domain, without qualitatively altering the resulting maps.

\section{Carpet plots and PCA}

This part of the analysis was performed using in-house python code, which we have made publicly available through GitHub (https://github.com/niksirbi/pcarpet/) and archived on Zenodo (doi: 10.5281/zenodo.5545696). We imported the preprocessed fMRI time series into python and computed the mean and standard deviation images across time, as well as their ratio (temporal signalto-noise ratio, or tSNR). Voxels with $\mathrm{tSNR}<15$ were omitted from further analysis. We then retained only cortical voxels (using the species-specific cortical masks from the aforementioned templates) and reshaped the data from 4D to a 2D matrix (voxels $x$ frames), with each voxel time series (row) normalized to zero mean and unit variance (z-score). To make global signal fluctuations more apparent on the 2D carpet plot, we reordered the voxels according to decreasing correlation with the mean cortical time series (Aquino et al., 2020). We then performed PCA on the carpet matrix using singular value decomposition. We extracted the first five temporal PCs and correlated them with all retained cortical voxels (Pearson's $r$ ). If a PCs' cortex-wide median $r$ value was negative, we signflipped the PC and its correlation values, to force the PC polarity to match the polarity of BOLD signal 
fluctuations for most voxels. We visualized the correlation values as heatmaps and as histograms per PC (50 bins between -1 and 1). The PCs with cortex-wide median $r$ value above a certain speciesspecific threshold (human 0.45 , macaque 0.15 , marmoset 0.22 , rat 0.2 ) were classified as 'asymmetric PCs'. The threshold was selected so that at most one prominent PC would exceed them for each fMRI run, with other PCs far below the threshold (see Fig. 1, 3, 5, 6 and Fig. S3). A few fMRI runs in the Rat 1 dataset had more than one PC close to or above the threshold and were thus excluded from downstream analysis (Fig 6b).

\section{Correspondence to EEG}

The human EEG acquisition and preprocessing has been described in past manuscripts (Golkowski et al., 2017; Ranft et al., 2016). As reported (Golkowski et al., 2017), fMRI volumes with low EEG power across all frequencies were classified as suppressions and assigned value 0 . Non-suppression epochs, which displayed high amplitude activity mainly in the delta band, were designated as bursts and assigned value 1 (see example in Fig. 1A). This process was performed for all anesthetized EEG-fMRI recordings-not just for the ones acquired with the high sevoflurane concentration (as in the previous analysis, Golkowski et al., 2017), which led us to discover that four intermediateconcentration recordings also contained suppression epochs. Recordings in which no fMRI volume was dominated by suppressed EEG power were classified as being in a continuous slow wave state.

For runs defined as burst-suppression by EEG, we convolved the boxcar envelope (bursts 1, suppression 0) with a canonical two-gamma hemodynamic response function (Glover, 1999) and applied the same bandpass filter as for fMRI preprocessing (0.005-0.12 Hz, see Fig. 1B). To compare the resulting EEG-derived hemodynamic model of burst-suppression with the asymmetric PC derived from the simultaneous fMRI recording, we computed their cross-correlation. We extracted each pair's correlation at zero lag, their maximum cross-correlation, and the time lag at which the maximum was achieved. Two runs acquired with the high sevoflurane concentration were excluded from the crosscorrelation analysis: subject 15 (EEG contained only one short burst and fMRI contained no asymmetric PC) and subject 7 (burst-suppression could be seen on EEG, but part of the recording was corrupted).

\section{Burst-suppression maps}

For each fMRI run with an asymmetric PC, we used the PC as a regressor in a first-level general linear model (GLM) analysis to get its distribution across the brain. The GLM was carried out with FEAT (FMRI Expert Analysis Tool Version 6.00, part of FSL), using local autocorrelation correction (Woolrich et al., 2001). Resulting $Z$ statistic images were masked for the brain and thresholded nonparametrically using clusters determined by $Z>3.1$ and a corrected cluster significance threshold of $P=0.05$ (Worsley, 2001). In the case of the human dataset, we also conducted the same analysis using 
the EEG-derived hemodynamic models as regressors. We compared the unthresholded Z statistic maps resulting from the two analyses by computing their spatial cross-correlation (ANTs MeasureImageSimilarity, neighborhood cross-correlation metric within the brain mask).

For each of the four species, we computed group-level burst-suppression maps using the resulting parameter estimate images from first-level GLMs. For subjects having more than one fMRI run with asymmetric PCs, the images were first averaged within each subject. Subsequently, a non-parametric one sample T-test was computed on the list of subject-level images, using FSL randomise (Winkler et al., 2014) with threshold-free cluster enhancement (TFCE, Smith and Nichols, 2009). The permutation-based randomise algorithm allows for control of false positives with minimal assumptions about the data, while TFCE has the sensitivity benefits of cluster-based thresholdingwithout the need for arbitrary decisions on cluster-forming thresholds. We thought these advantages to be important, given our goal to compare maps across dissimilar datasets, with varying spatial scales and signal-to-noise ratios. The resulting unthresholded group maps are given in Fig. S4 across species. Areas with a corrected $P<0.05$ were judged to be significantly correlated with asymmetric PCs. Significant anti-correlations were computed by repeating the above procedure with a flipped contrast. The thresholded maps are shown in Figs. 2 - 7. All group statistics were carried out in the species-specific volumetric template spaces. The resampling on cortical surfaces was done for purposes of data visualization only, using the methods described in the 'Template and atlases' paragraph.

\section{Region-of-interest analysis}

We defined two regions-of-interest per species-one in the left V1 and another in the left cingulate cortex (Cg: human area 24cd, macaque area 24c, marmoset area 24, rat primary cingulate cortex; see Fig. 8). The former was chosen because of its exclusion from primate burst-suppression maps; the latter because of its strong correlation with burst-suppression across species. We extracted each region's average BOLD signal time series from all preprocessed fMRI runs with asymmetric PCs and normalized them as percent-signal-change relative to the temporal mean. We quantified the standard deviation (SD) of the normalized time series as a measure of BOLD signal amplitude. For each species, we compared the BOLD SD between the two regions using a paired samples two-sided Wilcoxon ranksum test (jamovi v1.8 statistical software). We also computed the power spectral density (PSD) of the normalized time series using Welch's method (as implemented in scipy.signal.welch, segments of 100 timepoints, with 50 overlapping points between segments). Finally, each normalized time series was used as a regressor in a seed-based GLM analysis. The GLM statistics and thresholding were carried out exactly as described above for burst-suppression maps. A total of 8 seed-based group-level analyses were carried out ( 4 species x 2 seeds). 
In the human dataset, we further examined whether the BOLD signal SD and PSD of the two regions differed significantly between the two EEG-defined states. We focused on 12 human subjects, in which the EEG showed clear burst-suppression at the high sevoflurane concentration (3.9-4.6\%), and continuous slow waves at both the intermediate (3\%) and the low (2\%) sevoflurane concentrations. For each of V1 and cingulate seeds, we examined the effects of sevoflurane concentration on three response variables: BOLD signal amplitude (SD), low-frequency $\log _{10}$ PSD (integrated between 0.005$0.05 \mathrm{~Hz}$ ), and high-frequency $\log _{10}$ PSD (integrated between $0.05-0.12 \mathrm{~Hz}$ ). The lower frequency range corresponded to the timescale of burst-suppression in the human dataset. The effect of sevoflurane concentration on each of the three response variables was probed with repeated-measures ANOVA (sevoflurane concentration as the within-subject repeated-measures factor, jamovi v1.8, see Table S1). The Greenhouse-Geisser sphericity correction was applied when needed (Mauchly's sphericity test with $P<0.05)$. In cases of significant ANOVA effects $(P<0.05)$, we performed Tukey post-hoc pairwise comparisons between sevoflurane concentration levels (jamovi 1.8, see Table S2). Least Squares Means were estimated for the three levels of sevoflurane concentration. Results are summarized in Fig. S6. 


\section{References}

Aedo-Jury F, Schwalm M, Hamzehpour L, Stroh A. 2020. Brain states govern the spatio-temporal dynamics of resting-state functional connectivity. Elife 9:e53186. doi:10.7554/eLife.53186

Akrawi WP, Drummond JC, Kalkman CJ, Patel PM. 1996. A comparison of the electrophysiologic characteristics of EEG burst-suppression as produced by isoflurane, thiopental, etomidate, and propofol. J Neurosurg Anesthesiol 8:40-46.

An J, Jonnalagadda D, Moura V, Purdon PL, Brown EN, Westover MB. 2015. Spatial variation in automated burst suppression detection in pharmacologically induced coma. IEEE Eng Med Biol Soc Annu Conf 2015:7430-3. doi:10.1109/EMBC.2015.7320109

Aquino KM, Fulcher BD, Parkes L, Sabaroedin K, Fornito A. 2020. Identifying and removing widespread signal deflections from fMRI data: Rethinking the global signal regression problem. Neuroimage 212:116614. doi:https://doi.org/10.1016/j.neuroimage.2020.116614

Avants BB, Epstein CL, Gossman M, Gee JC. 2008. Symmetric diffeomorphic image registration with cross-correlation: Evaluating automated labeling of elderly and neurodegenerative brain. Med Image Anal 12:26-41. doi:10.1016/j.media.2007.06.004

Avants BB, Tustison NJ, Song G, Cook PA, Klein A, Gee JC. 2011. A reproducible evaluation of ANTs similarity metric performance in brain image registration. Neuroimage 54:2033-2044. doi:10.1016/j.neuroimage.2010.09.025

Avants BB, Yushkevich P, Pluta J, Minkoff D, Korczykowski M, Detre J, Gee JC. 2010. The optimal template effect in hippocampus studies of diseased populations. Neuroimage 49:2457-2466. doi:10.1016/j.neuroimage.2009.09.062

Barrière DA, Magalhães R, Novais A, Marques P, Selingue E, Geffroy F, Marques F, Cerqueira J, Sousa JC, Boumezbeur F, Bottlaender M, Jay TM, Cachia A, Sousa N, Mériaux S. 2019. The SIGMA rat brain templates and atlases for multimodal MRI data analysis and visualization. Nat Commun 10:1-13. doi:10.1038/s41467-019-13575-7

Bojak I, Stoyanov Z V., Liley DTJ. 2015. Emergence of spatially heterogeneous burst suppression in a neural field model of electrocortical activity. Front Syst Neurosci 9:18. doi:10.3389/fnsys.2015.00018

Brenner RP. 1985. The electroencephalogram in altered states of consciousness. Neurol Clin. doi:10.1016/s0733-8619(18)31025-9

Brown EN, Lydic R, Schiff ND. 2010. General anesthesia, sleep, and coma. N Engl J Med 363:26382650. doi:10.1056/NEJMra0808281

Brown EN, Purdon PL, van Dort CJ. 2011. General anesthesia and altered states of arousal: a systems neuroscience analysis. Annu Rev Neurosci 34:601-628. doi:10.1146/annurev-neuro-060909-153200

Ching S, Purdon PL, Vijayan S, Kopell NJ, Brown EN. 2012. A neurophysiological-metabolic model for burst suppression. Proc Natl Acad Sci U S A 109:3095-100. doi:10.1073/pnas.1121461109

Clark DL, Rosner BS. 1973. Neurophysiologic effects of general anesthetics. I. The electroencephalogram and sensory evoked responses in man. Anesthesiology. 


\section{doi:10.1097/00000542-197306000-00011}

Cloostermans MC, van Meulen FB, Eertman CJ, Hom HW, van Putten MJAM. 2012. Continuous electroencephalography monitoring for early prediction of neurological outcome in postanoxic patients after cardiac arrest. Crit Care Med 40:2867-2875. doi:10.1097/CCM.0b013e31825b94f0

Cox R. 1996. AFNI: software for analysis and visualization of functional magnetic resonance neuroimages. Comput Biomed Res 29:162-173. doi:10.1006/CBMR.1996.0014

Detsch O, Kochs E, Siemers M, Bromm B, Vahle-Hinz C. 2002. Increased responsiveness of cortical neurons in contrast to thalamic neurons during isoflurane-induced EEG bursts in rats. Neurosci Lett 317:9-12. doi:10.1016/S0304-3940(01)02419-3

Essen DC Van, Donahue CJ, Coalson TS, Kennedy H, Hayashi T, Glasser MF. 2019. Cerebral cortical folding, parcellation, and connectivity in humans, nonhuman primates, and mice. Proc Natl Acad Sci 116:26173-26180. doi:10.1073/PNAS.1902299116

Fischl B. 2012. FreeSurfer. Neuroimage 62:774-781. doi:10.1016/J.NEUROIMAGE.2012.01.021

Fischl B, Sereno MI, Tootell RBH, Dale AM. 1999. High-resolution intersubject averaging and a coordinate system for the cortical surface. Hum Brain Mapp 8:272-284. doi:10.1002/(SICI)10970193(1999)8:4<272::AID-HBM10>3.0.C0;2-4

Fleischmann A, Pilge S, Kiel T, Kratzer S, Schneider G, Kreuzer M. 2018. Substance-Specific Differences in Human Electroencephalographic Burst Suppression Patterns. Front Hum Neurosci 12:368. doi:10.3389/fnhum.2018.00368

Froudist-Walsh S, Xu T, Niu M, Rapan L, Margulies DS, Zilles K, Wang X-J, Palomero-Gallagher N. 2021. Gradients of receptor expression in the macaque cortex. bioRxiv 2021.02.22.432173. doi:10.1101/2021.02.22.432173

Fulcher BD, Murray JD, Zerbi V, Wang X-J. 2019. Multimodal gradients across mouse cortex. Proc Natl Acad Sci 116:4689-4695. doi:10.1073/PNAS.1814144116

Fultz NE, Bonmassar G, Setsompop K, Stickgold RA, Rosen BR, Polimeni JR, Lewis LD. 2019. Coupled electrophysiological, hemodynamic, and cerebrospinal fluid oscillations in human sleep. Science (80) 366:628 LP - 631. doi:10.1126/science.aax5440

Gao Y-RR, Ma Y, Zhang Q, Winder AT, Liang Z, Antinori L, Drew PJ, Zhang N. 2017. Time to wake up: Studying neurovascular coupling and brain-wide circuit function in the un-anesthetized animal. Neuroimage 153:382-398. doi:10.1016/j.neuroimage.2016.11.069

Glasser MF, Coalson TS, Robinson EC, Hacker CD, Harwell J, Yacoub E, Ugurbil K, Andersson J, Beckmann CF, Jenkinson M, Smith SM, van Essen DC. 2016. A multi-modal parcellation of human cerebral cortex. Nature 536:171-178. doi:10.1038/nature18933

Glover GH. 1999. Deconvolution of Impulse Response in Event-Related BOLD fMRI1. Neuroimage 9:416-429. doi:10.1006/NIMG.1998.0419

Golkowski D, Larroque S, Vanhaudenhuyse A, Plenevaux A, Boly M, Di Perri C, Ranft A, Schneider G, Laureys S, Jordan D, Bonhomme V, Ilg R. 2019. Changes in Whole Brain Dynamics and Connectivity Patterns during Sevoflurane- and Propofol-induced Unconsciousness Identified by Functional 
Magnetic Resonance Imaging. Anesthesiology 130:898-911. doi:10.1097/ALN.0000000000002704

Golkowski D, Ranft A, Kiel T, Riedl V, Kohl P, Rohrer G, Pientka J, Berger S, Preibisch C, Zimmer C, Mashour GA, Schneider G, Kochs EF, Ilg R, Jordan D. 2017. Coherence of BOLD signal and electrical activity in the human brain during deep sevoflurane anesthesia. Brain Behav 7:e00679. doi:10.1002/brb3.679

Gorgolewski K, Burns CD, Madison C, Clark D, Halchenko YO, Waskom ML, Ghosh SS. 2011. Nipype: A Flexible, Lightweight and Extensible Neuroimaging Data Processing Framework in Python. Front Neuroinform 5:13. doi:10.3389/fninf.2011.00013

Grabner G, Janke AL, Budge MM, Smith D, Pruessner J, Collins DL. 2006. Symmetric atlasing and model based segmentation: An application to the hippocampus in older adultsLecture Notes in Computer Science (Including Subseries Lecture Notes in Artificial Intelligence and Lecture Notes in Bioinformatics). Springer Verlag. pp. 58-66. doi:10.1007/11866763_8

Hartig R, Glen D, Jung B, Logothetis NK, Paxinos G, Garza-Villarreal EA, Messinger A, Evrard HC. 2021. The Subcortical Atlas of the Rhesus Macaque (SARM) for neuroimaging. Neuroimage 235:117996. doi:10.1016/J.NEUROIMAGE.2021.117996

Hartikainen KM, Rorarius M, Peräkylä JJ, Laippala PJ, Jäntti V. 1995. Cortical reactivity during isoflurane burst-suppression anesthesia. Anesth Analg 81:1223-8. doi:10.1097/00000539199512000-00018

Hillman EMC. 2014. Coupling mechanism and significance of the BOLD signal: a status report. Annu Rev Neurosci 37:161-181. doi:10.1146/annurev-neuro-071013-014111

Hofmeijer J, Tjepkema-Cloostermans MC, van Putten MJAM. 2014. Burst-suppression with identical bursts: A distinct EEG pattern with poor outcome in postanoxic coma. Clin Neurophysiol 125:947954. doi:10.1016/j.clinph.2013.10.017

Hudetz AG, Imas OA. 2007. Burst activation of the cerebral cortex by flash stimuli during isoflurane anesthesia in rats. Anesthesiology 107:983-991. doi:10.1097/01.anes.0000291471.80659.55

Huntenburg JM, Yeow LY, Mandino F, Grandjean J. 2021. Gradients of functional connectivity in the mouse cortex reflect neocortical evolution. Neuroimage 225:117528. doi:10.1016/J.NEUROIMAGE.2020.117528

Jenkinson M, Beckmann CF, Behrens TEJ, Woolrich MW, Smith SM. 2012. FSL. Neuroimage 62:782790. doi:10.1016/J.NEUROIMAGE.2011.09.015

Jung B, Taylor PA, Seidlitz J, Sponheim C, Perkins P, Ungerleider LG, Glen D, Messinger A. 2021. A comprehensive macaque fMRI pipeline and hierarchical atlas. Neuroimage 235:117997. doi:10.1016/j.neuroimage.2021.117997

Kalthoff D, Po C, Wiedermann D, Hoehn M. 2013. Reliability and spatial specificity of rat brain sensorimotor functional connectivity networks are superior under sedation compared with general anesthesia. NMR Biomed 26:638-650. doi:10.1002/nbm.2908

Kroeger D, Amzica F. 2007. Hypersensitivity of the anesthesia-induced comatose brain. J Neurosci 27:10597-10607. doi:10.1523/JNEUROSCI.3440-07.2007 
Kroeger D, Florea B, Amzica F. 2013. Human brain activity patterns beyond the isoelectric line of extreme deep coma. PLoS One 8:e75257. doi:10.1371/journal.pone.0075257

Land R, Engler G, Kral A, Engel AK. 2012. Auditory Evoked Bursts in Mouse Visual Cortex during Isoflurane Anesthesia. PLoS One 7:49855. doi:10.1371/JOURNAL.PONE.0049855

Lewis LD, Ching SN, Weiner VS, Peterfreund RA, Eskandar EN, Cash SS, Brown EN, Purdon PL. 2013. Local cortical dynamics of burst suppression in the anaesthetized brain. Brain 136:2727-2737. doi:10.1093/brain/awt174

Li X, Morgan P, Ashburner J, Smith J, Rorden C. 2016. The first step for neuroimaging data analysis: DICOM to NIfTI conversion. J Neurosci Methods 264:47-56. doi:10.1016/J.JNEUMETH.2016.03.001

Liley DTJ, Walsh M. 2013. The Mesoscopic Modeling of Burst Suppression during Anesthesia. Front Comput Neurosci 7:46. doi:10.3389/fncom.2013.00046

Liu C, Ye FQ, Yen CCC, Newman JD, Glen D, Leopold DA, Silva AC. 2018. A digital 3D atlas of the marmoset brain based on multi-modal MRI. Neuroimage 169:106-116. doi:10.1016/j.neuroimage.2017.12.004

Liu C, Yen CCC, Szczupak D, Tian X, Glen D, Silva AC. 2021. Marmoset Brain Mapping V3: Population multi-modal standard volumetric and surface-based templates. Neuroimage 226:117620. doi:10.1016/j.neuroimage.2020.117620

Liu X, Zhu X-H, Zhang Y, Chen W. 2011. Neural origin of spontaneous hemodynamic fluctuations in rats under burst-suppression anesthesia condition. Cereb Cortex 21:374-384. doi:10.1093/cercor/bhq105

Liu X, Zhu XH, Zhang Y, Chen W. 2013. The change of functional connectivity specificity in rats under various anesthesia levels and its neural origin. Brain Topogr 26:363-377. doi:10.1007/s10548-0120267-5

Logothetis NK, Pfeuffer J. 2004. On the nature of the BOLD fMRI contrast mechanism. Magn Reson Imaging 22:1517-1531. doi:10.1016/j.mri.2004.10.018

Lukatch HS, Kiddoo CE, Maciver MB. 2005. Anesthetic-induced burst suppression EEG activity requires glutamate-mediated excitatory synaptic transmission. Cereb Cortex 15:1322-1331. doi:10.1093/cercor/bhi015

Lukatch HS, MacIver BM. 1996. Synaptic Mechanisms of Thiopental-induced Alterations in Synchronized Cortical Activity. Anesthesiol J Am Soc Anesthesiol 84:1425-1434. doi:0000542199606000-00019

Mandino F, Cerri DH, Garin CM, Straathof M, van Tilborg GAF, Chakravarty MM, Dhenain M, Dijkhuizen RM, Gozzi A, Hess A, Keilholz SD, Lerch JP, Shih Y-YI, Grandjean J. 2020. Animal Functional Magnetic Resonance Imaging: Trends and Path Toward Standardization . Front Neuroinformatics .

Marcus D, Harwell J, Olsen T, Hodge M, Glasser M, Prior F, Jenkinson M, Laumann T, Curtiss S, Van Essen D. 2011. Informatics and Data Mining Tools and Strategies for the Human Connectome Project. Front Neuroinform 0:4. doi:10.3389/FNINF.2011.00004

Margulies DS, Ghosh SS, Goulas A, Falkiewicz M, Huntenburg JM, Langs G, Bezgin G, Eickhoff SB, 
Castellanos FX, Petrides M, Jefferies E, Smallwood J. 2016. Situating the default-mode network along a principal gradient of macroscale cortical organization. Proc Natl Acad Sci U S A 113:12574-12579. doi:10.1073/pnas.1608282113

Masamoto K, Fukuda M, Vazquez A, Kim S-G. 2009. Dose-dependent effect of isoflurane on neurovascular coupling in rat cerebral cortex. Eur J Neurosci 30:242-250. doi:10.1111/j.14609568.2009.06812.x

Milham M, Petkov CI, Margulies DS, Schroeder CE, Basso MA, Belin P, Fair DA, Fox A, Kastner S, Mars RB, Messinger A, Poirier C, Vanduffel W, Essen DC Van, Alvand A, Becker Y, Hamed S Ben, Benn A, Bodin C, Boretius S, Cagna B, Coulon O, El-Gohary SH, Evrard H, Forkel SJ, Friedrich P, Froudist-Walsh S, Garza-Villarreal EA, Gao Y, Gozzi A, Grigis A, Hartig R, Hayashi T, Heuer K, Howells H, Ardesch DJ, Jarraya B, Jarrett W, Jedema HP, Kagan I, Kelly C, Kennedy H, Klink PC, Kwok SC, Leech R, Liu X, Madan C, Madushanka W, Majka P, Mallon A-M, Marche K, Meguerditchian A, Menon RS, Merchant H, Mitchell A, Nenning K-H, Nikolaidis A, Ortiz-Rios M, Pagani M, Pareek V, Prescott M, Procyk E, Rajimehr R, Rautu I-S, Raz A, Roe AW, Rossi-Pool R, Roumazeilles L, Sakai T, Sallet J, García-Saldivar P, Sato C, Sawiak S, Schiffer M, Schwiedrzik CM, Seidlitz J, Sein J, Shen Z, Shmuel A, Silva AC, Simone L, Sirmpilatze N, Sliwa J, Smallwood J, Tasserie J, de Schotten MT, Toro R, Trapeau R, Uhrig L, Vezoli J, Wang Z, Wells S, Williams B, Xu T, Xu AG, Yacoub E, Zhan M, Ai L, Amiez C, Balezeau F, Baxter MG, Blezer ELA, Brochier T, Chen A, Croxson PL, Damatac CG, Dehaene S, Everling S, Fleysher L, Freiwald W, Griffiths TD, Guedj C, Hadj-Bouziane F, Harel N, Hiba B, Jung B, Koo B, Laland KN, Leopold DA, Lindenfors P, Meunier M, Mok K, Morrison JH, Nacef J, Nagy J, Pinsk M, Reader SM, Roelfsema PR, Rudko DA, Rushworth MFS, Russ BE, Schmid MC, Sullivan EL, Thiele A, Todorov OS, Tsao D, Ungerleider L, Wilson CRE, Ye FQ, Zarco W, Zhou Y. 2020. Accelerating the Evolution of Nonhuman Primate Neuroimaging. Neuron 105:600-603. doi:https://doi.org/10.1016/j.neuron.2019.12.023

Ming Q, Liou JY, Yang F, Li J, Chu C, Zhou Q, Wu D, Xu S, Luo P, Liang J, Li D, Pryor KO, Lin W, Schwartz $\mathrm{TH}, \mathrm{Ma}$ H. 2021. Isoflurane-Induced Burst Suppression Is a Thalamus-Modulated, Focal-Onset Rhythm With Persistent Local Asynchrony and Variable Propagation Patterns in Rats. Front Syst Neurosci 14:102. doi:10.3389/fnsys.2020.599781

Muller L, Chavane F, Reynolds J, Sejnowski TJ. 2018. Cortical travelling waves: mechanisms and computational principles. Nat Rev Neurosci 19:255-268. doi:10.1038/nrn.2018.20

Ogawa S, Lee TM, Kay AR, Tank DW. 1990. Brain magnetic resonance imaging with contrast dependent on blood oxygenation. Proc Natl Acad Sci 87:9868-9872. doi:10.1073/pnas.87.24.9868

Paasonen J, Laakso H, Pirttimäki T, Stenroos P, Salo RA, Zhurakovskaya E, Lehto LJ, Tanila H, Garwood M, Michaeli S, Idiyatullin D, Mangia S, Gröhn O. 2019. Multi-band SWIFT enables quiet and artefactfree EEG-fMRI and awake fMRI studies in rat. Neuroimage 116338. doi:10.1016/J.NEUROIMAGE.2019.116338

Paasonen J, Salo RA, Shatillo A, Forsberg MM, Närväinen J, Huttunen JK, Gröhn O. 2016. Comparison of seven different anesthesia protocols for nicotine pharmacologic magnetic resonance imaging in rat. Eur Neuropsychopharmacol 26:518-531. doi:10.1016/j.euroneuro.2015.12.034

Paasonen J, Stenroos P, Salo RA, Kiviniemi V, Gröhn O. 2018. Functional connectivity under six anesthesia protocols and the awake condition in rat brain. Neuroimage 172:9-20. doi:10.1016/J.NEUROIMAGE.2018.01.014

Pilge S, Jordan D, Kreuzer M, Kochs EF, Schneider G. 2014. Burst suppression-MAC and burst 
suppression-CP(5)(0) as measures of cerebral effects of anaesthetics. Br J Anaesth 112:1067-1074. doi:10.1093/bja/aeu016

Power JD. 2017. A simple but useful way to assess fMRI scan qualities. Neuroimage 154:150-158. doi:10.1016/J.NEUROIMAGE.2016.08.009

Ranft A, Golkowski D, Kiel T, Riedl V, Kohl P, Rohrer G, Pientka J, Berger S, Thul A, Maurer M, Preibisch C, Zimmer C, Mashour GA, Kochs EF, Jordan D, Ilg R. 2016. Neural Correlates of Sevoflurane-induced Unconsciousness Identified by Simultaneous Functional Magnetic Resonance Imaging and Electroencephalography. Anesthesiology 125:861-872. doi:10.1097/ALN.0000000000001322

Sanchez-Vives M V., Massimini M, Mattia M. 2017. Shaping the Default Activity Pattern of the Cortical Network. Neuron. doi:10.1016/j.neuron.2017.05.015

Schwalm M, Schmid F, Wachsmuth L, Backhaus H, Kronfeld A, Aedo Jury F, Prouvot P-H, Fois C, Albers F, van Alst T, Faber C, Stroh A. 2017. Cortex-wide BOLD fMRI activity reflects locally-recorded slow oscillation-associated calcium waves. Elife 6. doi:10.7554/eLife.27602

Seidlitz J, Sponheim C, Glen D, Ye FQ, Saleem KS, Leopold DA, Ungerleider L, Messinger A. 2018. A population MRI brain template and analysis tools for the macaque. Neuroimage 170:121-131. doi:10.1016/J.NEUROIMAGE.2017.04.063

Sirmpilatze N, Baudewig J, Boretius S. 2019. Temporal stability of fMRI in medetomidine-anesthetized rats. Sci Rep 9. doi:10.1038/s41598-019-53144-y

Smith SM, Nichols TE. 2009. Threshold-free cluster enhancement: Addressing problems of smoothing, threshold dependence and localisation in cluster inference. Neuroimage 44:83-98. doi:10.1016/j.neuroimage.2008.03.061

Stenroos P, Pirttimäki T, Paasonen J, Paasonen E, Salo RA, Koivisto H, Natunen T, Mäkinen P, Kuulasmaa T, Hiltunen M, Tanila H, Gröhn 0. 2021. Isoflurane affects brain functional connectivity in rats 1 month after exposure. Neuroimage 234:117987. doi:10.1016/j.neuroimage.2021.117987

Steriade M, Amzica F, Contreras D. 1994. Cortical and thalamic cellular correlates of electroencephalographic burst-suppression. Electroencephalogr Clin Neurophysiol 90:1-16. doi:10.1016/0013-4694(94)90108-2

Steriade M, Nunez A, Amzica F. 1993. A novel slow ( $<1 \mathrm{~Hz}$ ) oscillation of neocortical neurons in vivo: Depolarizing and hyperpolarizing components. J Neurosci 13:3252-3265. doi:10.1523/jneurosci.1308-03252.1993

Stroh A, Adelsberger H, Groh A, Rühlmann C, Fischer S, Schierloh A, Deisseroth K, Konnerth A. 2013. Making waves: initiation and propagation of corticothalamic Ca2+ waves in vivo. Neuron 77:11361150. doi:10.1016/j.neuron.2013.01.031

Swank RL. 1949. Synchronization of spontaneous electrical activity of cerebrum by barbiturate narcosis. J Neurophysiol 12:161-172. doi:10.1152/jn.1949.12.3.161

Swank RL, Watson CW. 1949. Effects of barbiturates and ether on spontaneous electrical activity of dog brain. J Neurophysiol 12:137-160. doi:10.1152/jn.1949.12.2.137

Tustison NJ, Avants BB, Cook PA, Zheng Y, Egan A, Yushkevich PA, Gee JC. 2010. N4ITK: Improved N3 
bias correction. IEEE Trans Med Imaging. doi:10.1109/TMI.2010.2046908

Vincent JL, Patel GH, Fox MD, Snyder AZ, Baker JT, Van Essen DC, Zempel JM, Snyder LH, Corbetta M, Raichle ME. 2007. Intrinsic functional architecture in the anaesthetized monkey brain. Nature 447:83-86. doi:10.1038/nature05758

Westover MB, Ching S, Kumaraswamy VM, Akeju O, Pierce E, Cash SS, Kilbride R, Brown EN, Purdon PL. 2015. The human burst suppression electroencephalogram of deep hypothermia. Clin Neurophysiol 126:1901-1914. doi:10.1016/J.CLINPH.2014.12.022

Williams KA, Magnuson ME, Majeed W, LaConte SM, Peltier SJ, Hu X, Keilholz SD. 2010. Comparison of alpha-chloralose, medetomidine and isoflurane anesthesia for functional connectivity mapping in the rat. Magn Reson Imaging 28:995-1003. doi:10.1016/j.mri.2010.03.007

Winkler AM, Ridgway GR, Webster MA, Smith SM, Nichols TE. 2014. Permutation inference for the general linear model. Neuroimage 92:381-397. doi:10.1016/j.neuroimage.2014.01.060

Woolrich MW, Ripley BD, Brady M, Smith SM. 2001. Temporal Autocorrelation in Univariate Linear Modeling of FMRI Data. Neuroimage 14:1370-1386. doi:10.1006/NIMG.2001.0931

Worsley KJ. 2001. Statistical analysis of activation imagesFunctional Magnetic Resonance Imaging. Oxford University Press. pp. 251-270. doi:10.1093/acprof:oso/9780192630711.003.0014

Wu J, Ngo GH, Greve D, Li J, He T, Fischl B, Eickhoff SB, Yeo BTT. 2018. Accurate nonlinear mapping between MNI volumetric and FreeSurfer surface coordinate systems. Hum Brain Mapp 39:37933808. doi:10.1002/hbm.24213

Young GB. 2000. The EEG in coma. J Clin Neurophysiol 17:473-85.

Yushkevich PA, Piven J, Hazlett HC, Smith RG, Ho S, Gee JC, Gerig G. 2006. User-guided 3D active contour segmentation of anatomical structures: significantly improved efficiency and reliability. Neuroimage 31:1116-1128. doi:10.1016/j.neuroimage.2006.01.015

Zhang D, Ding H, Ye D, Jia X, Thakor N. 2010. Burst Suppression EEG during Hypothermia and Rapid Rewarming in Isoflurane-Anesthetized Rats2010 4th International Conference on Bioinformatics and Biomedical Engineering. IEEE. pp. 1-4. doi:10.1109/ICBBE.2010.5514818

Zhang Z, Cai D-C, Wang Zhiwei, Zeljic K, Wang Zheng, Wang Y. 2019. Isoflurane-Induced Burst Suppression Increases Intrinsic Functional Connectivity of the Monkey Brain. Front Neurosci 13:296. doi:10.3389/fnins.2019.00296 


\section{Supplementary Material}

Figure S1 Examples of fMRI runs without asymmetric PCs

Figure S2 Ventricular motion during burst-suppression

Figure S3 Identifying and mapping asymmetric PCs in the Rat 2 dataset

Figure S4 Unthresholded asymmetric PC maps across species

Figure S5 Temporal signal-to-noise (tSNR) maps across species

Figure S6 BOLD signal time series across sevoflurane concentrations in humans

Figure S7 Burst-suppression timescales across species

Table S1 Repeated-measures ANOVA results for Figure S6

Table S2 Post-hoc pairwise tests for Figure S6 
bioRxiv preprint doi: https://doi.org/10.1101/2021.10.15.464515; this version posted October 16, 2021. The copyright holder for this preprint (which was not certified by peer review) is the author/funder, who has granted bioRxiv a license to display the preprint in perpetuity. It is made available under aCC-BY 4.0 International license.

A
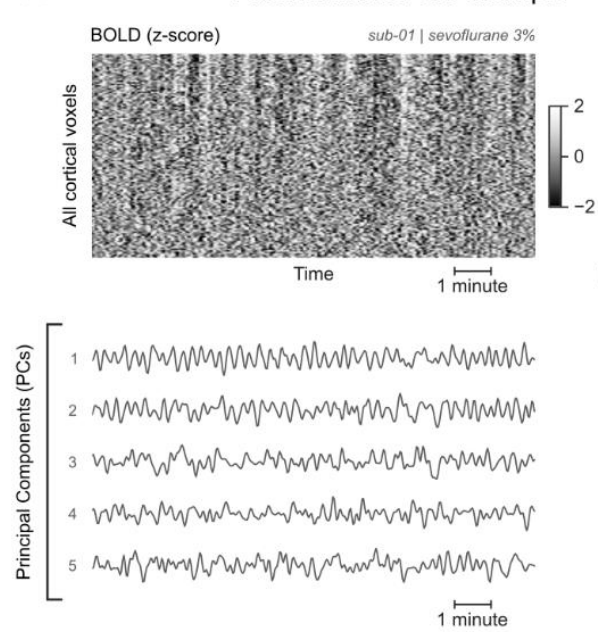

C

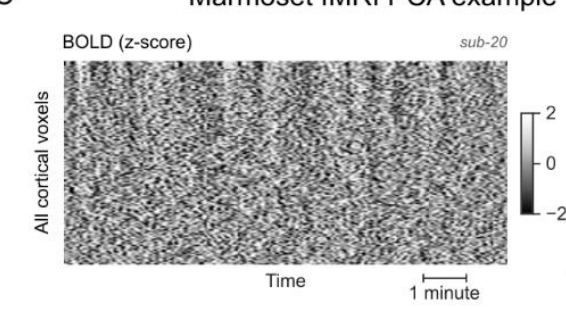

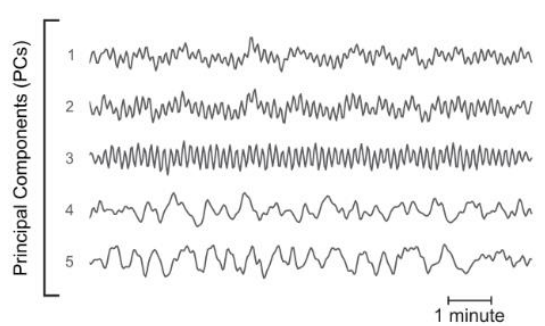
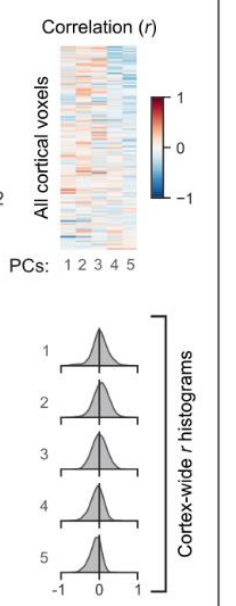

B Macaque fMRI PCA example

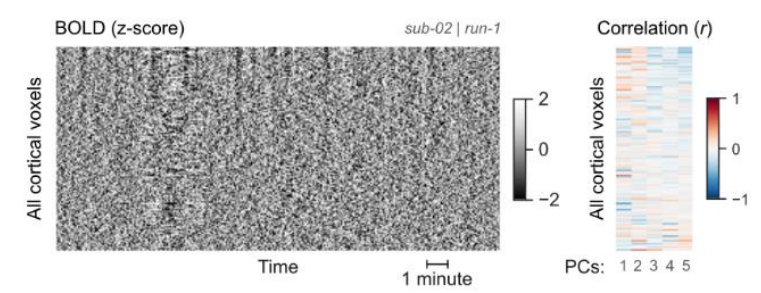

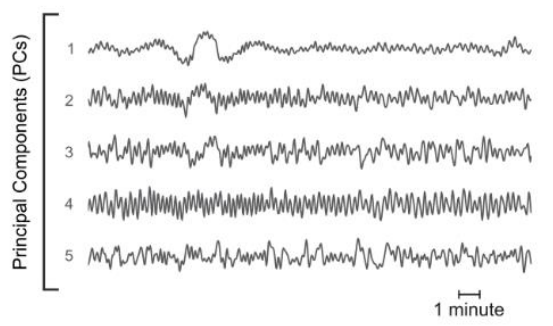

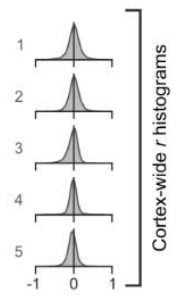

D
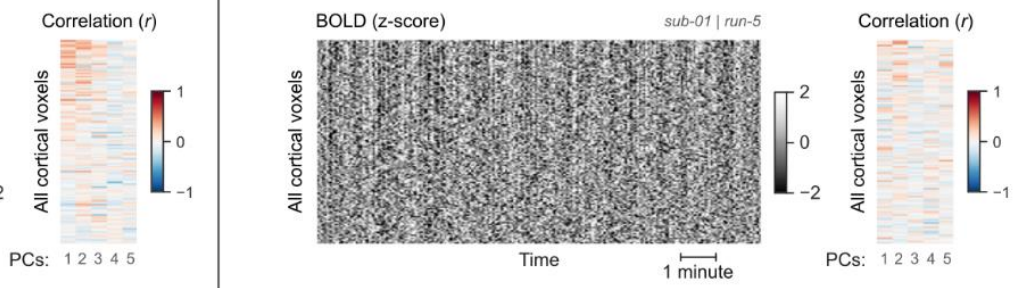

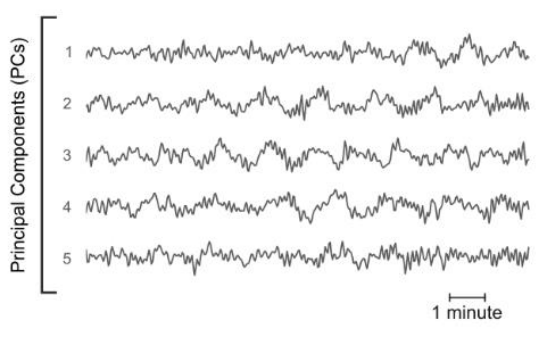

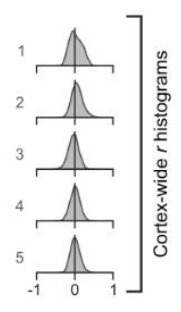

Figure S1. Examples of fMRI runs without asymmetric PCs. (A) Panel shows example data from a human participant anesthetized with $3 \%$ sevoflurane, while the other panels show examples from animal subjects anesthetized with isoflurane: (B) macaque, (C) marmoset, and (D) rat. For each example, the cortical BOLD fMRI signal is represented as a carpet plot. The rows (voxels) are ordered according to their correlation with the mean cortical signal to better visualize widespread fluctuations. The first five temporal PCs of the signal are plotted below the carpet plot. The Pearson's correlation coefficients $(r)$ between the PCs and all cortical voxels are represented both as a heatmap and as histograms for each PC. All PCs have symmetric histograms, centered around $r=0$. Related to Figs. 1, 3, 5, and 6 . 

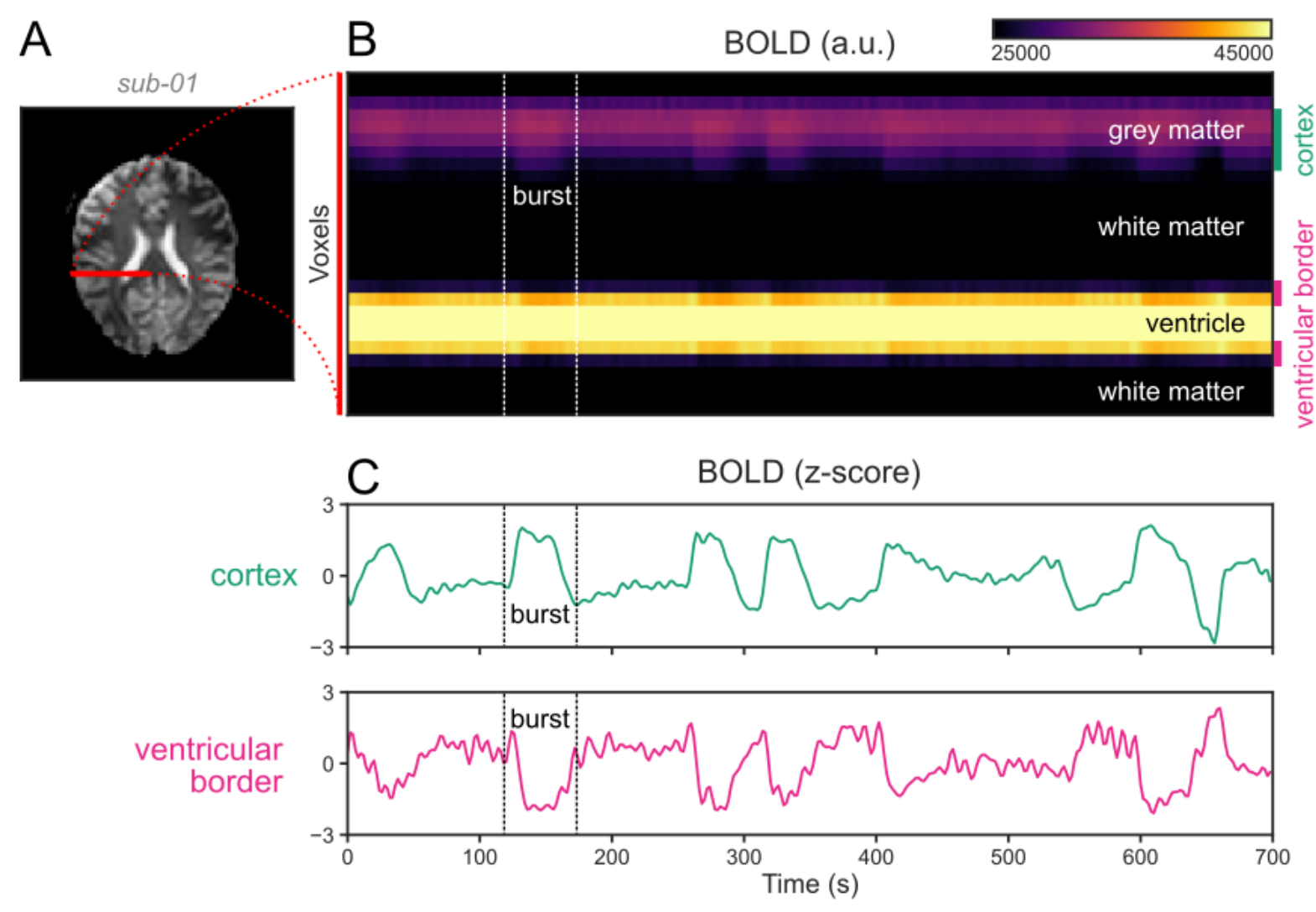

Figure S2. Ventricular motion during burst-suppression. (A) An axial slice from an echo planar imaging (EPI) BOLD fMRI scan of a human subject during burst-suppression (same run as in Fig. 1). A line profile is marked in red, stretching from the pial surface of the right hemisphere towards the midline, going through cortical grey matter, white matter, and the lateral ventricle. (B) The BOLD signal across this line profile is plotted for the entire duration of the fMRI run (700 s). One of the bursts is marked by vertical dashed lines. (C) During bursts, the mean BOLD signal of the cortical voxels rises-as expected, whereas the signal at the ventricular borders falls. This is most likely caused by an inward motion of the ventricular borders, with the low-intensity BOLD signal of the surrounding white matter replacing the higher-intensity ventricular signal. Even if the motion occurs on a sub-voxel scale, partial volume effects would still cause the darkening of the border. 
bioRxiv preprint doi: https://doi.org/10.1101/2021.10.15.464515; this version posted October 16, 2021. The copyright holder for this preprint (which was not certified by peer review) is the author/funder, who has granted bioRxiv a license to display the preprint in perpetuity. It is made available under aCC-BY 4.0 International license.

A

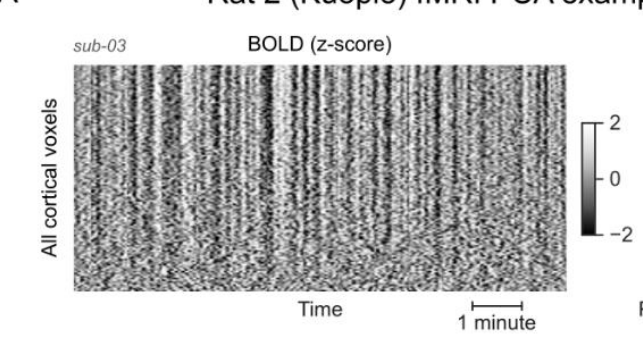

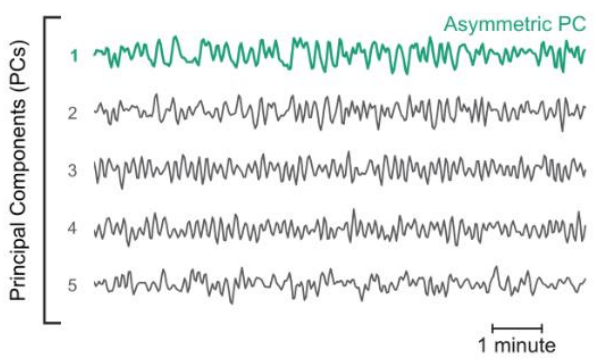

Correlation ( $r$ )
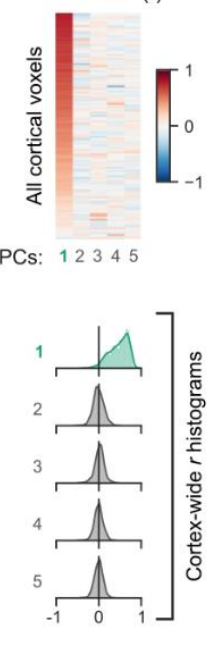

B

Identifying asymmetric PCs
Rat 2 dataset

isoflurane concentration: $1.3 \%$

- Asymmetric PC $(r>0.2) \quad \circ$ Other PCs

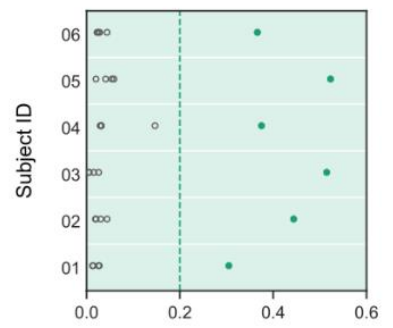

|Median| cortex-wide correlation ( $r$ ) with PC

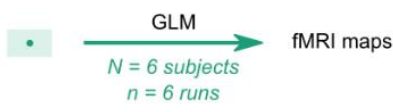

Asymmetric PC map (group)
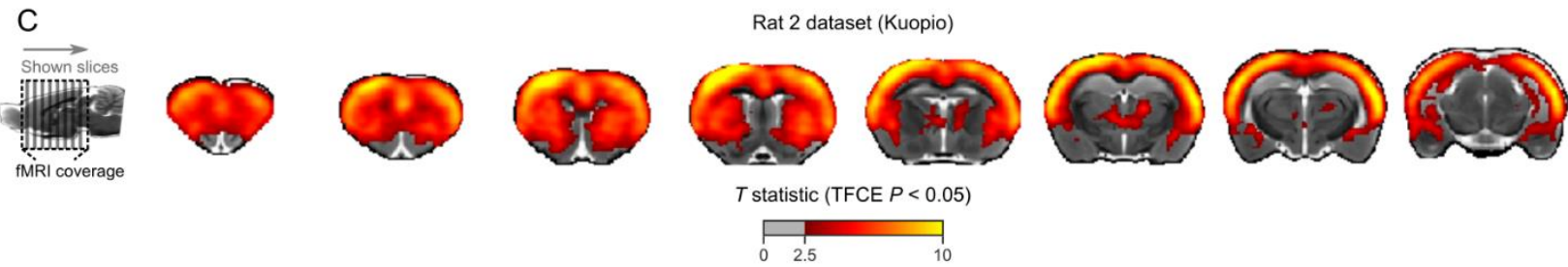

D
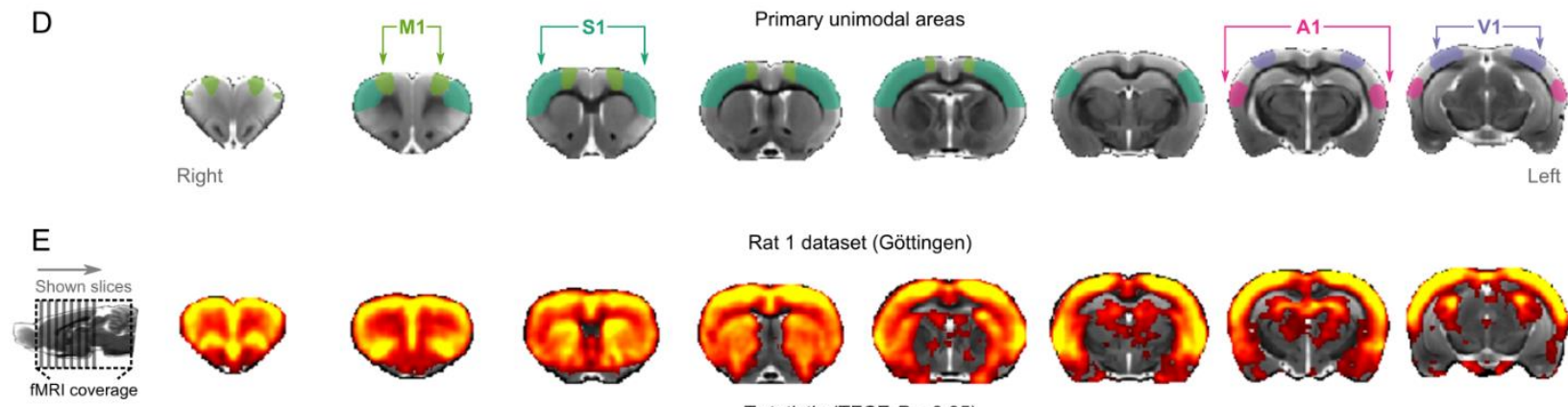

Rat 1 dataset (Göttingen)
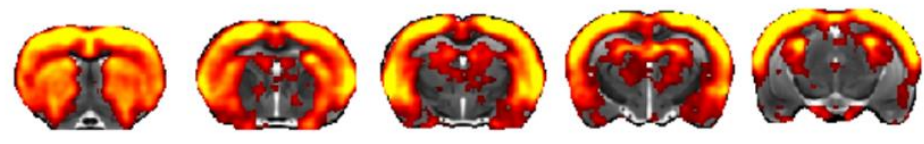

$T$ statistic (TFCE $P<0.05$ )

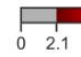

Figure S3. Identifying and mapping asymmetric PCs in the Rat 2 dataset. (A) The cortical BOLD fMRI signal of an isoflurane-anesthetized subject from the Rat 2 dataset is represented as a carpet plot. The first five temporal PCs of the signal are plotted below the carpet plot. The Pearson's correlation coefficients $(r)$ between the PCs and all cortical voxels are represented both as a heatmap and as histograms for each PC. The first PC captures the widespread fluctuation that is visible on the carpet plot and has an asymmetric $r$ histogram. (B) Cortex-wide median $r$ values for the first five PCs are plotted as dots across the entire Rat 2 dataset ( 6 animals, one fMRI run each). All fMRI runs have a prominent asymmetric PC ( $r>0.2$, highlighted in green) and are used as regressors for GLM analysis. (C) The group asymmetric PC map-computed via a second-level analysis of single-subject GLMs is shown overlaid on a study-specific volumetric template. The group statistics were carried out with FSL randomise; the resulting $T$ statistic maps were thresholded using Threshold-Free Cluster Enhancement (TFCE) and a corrected $P<0.05$. (D) The locations of primary motor (M1), somatosensory (S1), auditory (A1), and visual (V1) cortices-based on the SIGMA rat brain atlas-are indicated for reference. (E) The corresponding map of the Rat 1 dataset (the same as in Fig. 6C) is shown for comparison. Related to Fig. 6. 
bioRxiv preprint doi: https://doi.org/10.1101/2021.10.15.464515; this version posted October 16, 2021. The copyright holder for this preprint (which was not certified by peer review) is the author/funder, who has granted bioRxiv a license to display the preprint in perpetuity. It is made available under aCC-BY 4.0 International license.

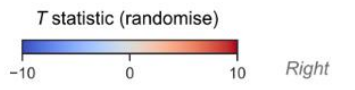

\section{A Atminn}

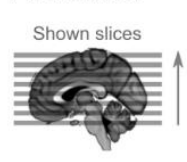

$N=19$ subjects
$n=21$ runs
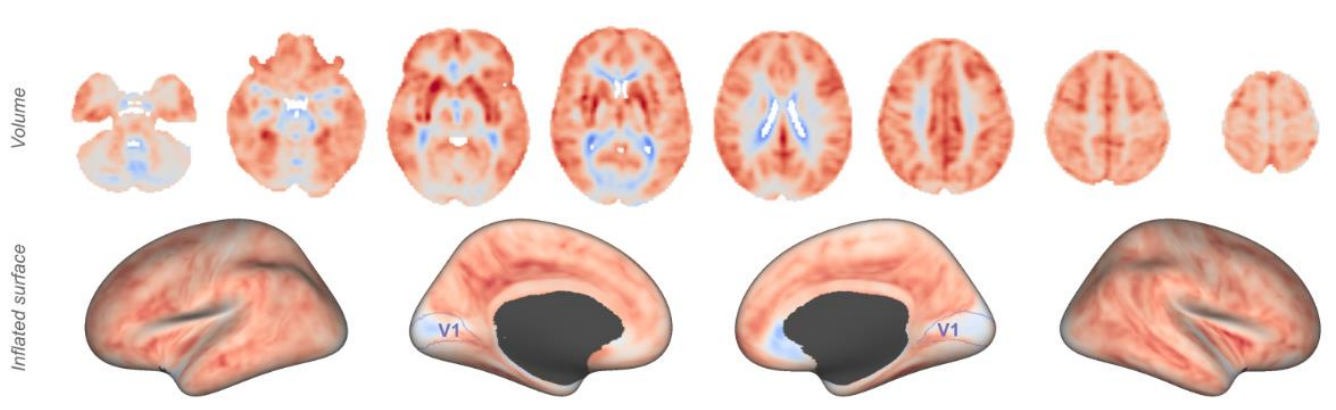

B. Macaque
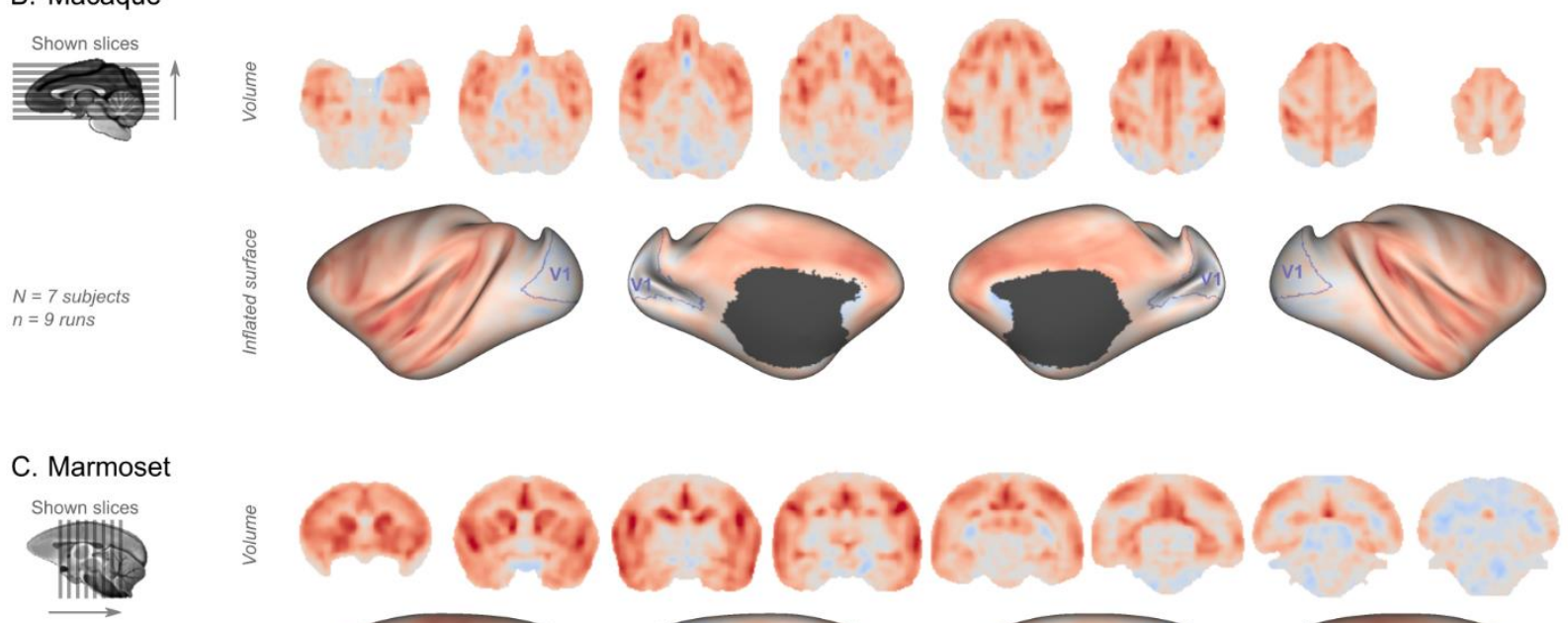

$$
\begin{aligned}
& N=8 \text { subjects } \\
& n=8 \text { runs }
\end{aligned}
$$
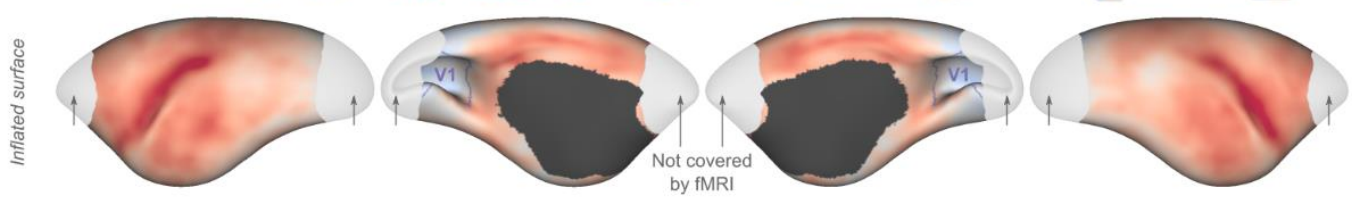

D. Rat
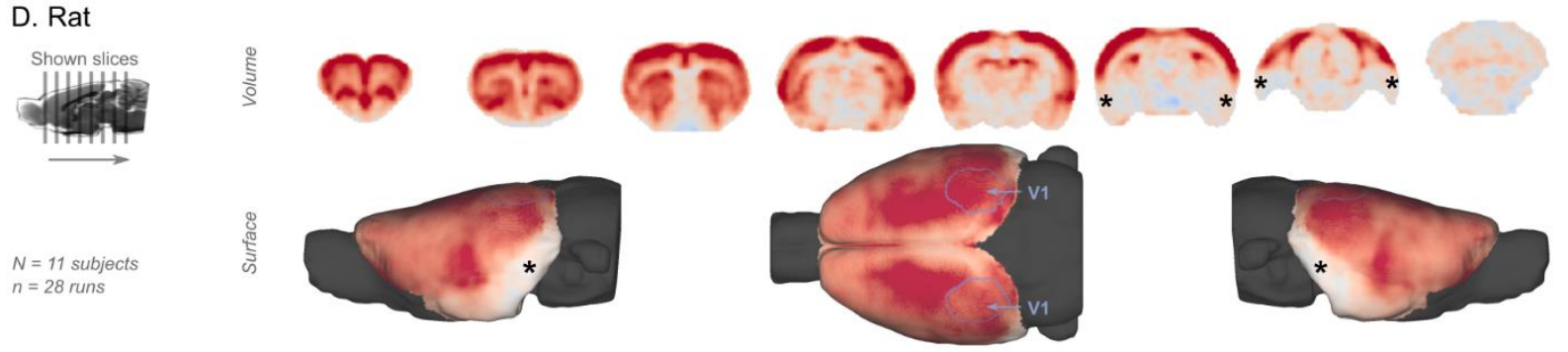

Figure S4. Unthresholded asymmetric PC maps across species. The unthresholded group-level $T$ statistic maps - (A) human, (B) macaque, (C) marmoset, (D) rat - are overlaid on volumetric slices and surfaces identical to the ones given in Figs. 2, 3, 5 and 6. The location of V1 for each species is marked by purple outlines. Asterisks on the rat map indicate areas with low signal-to-noise ratio (see also Fig. S5). Related to Figs. 2, 3, 5, and 6. 

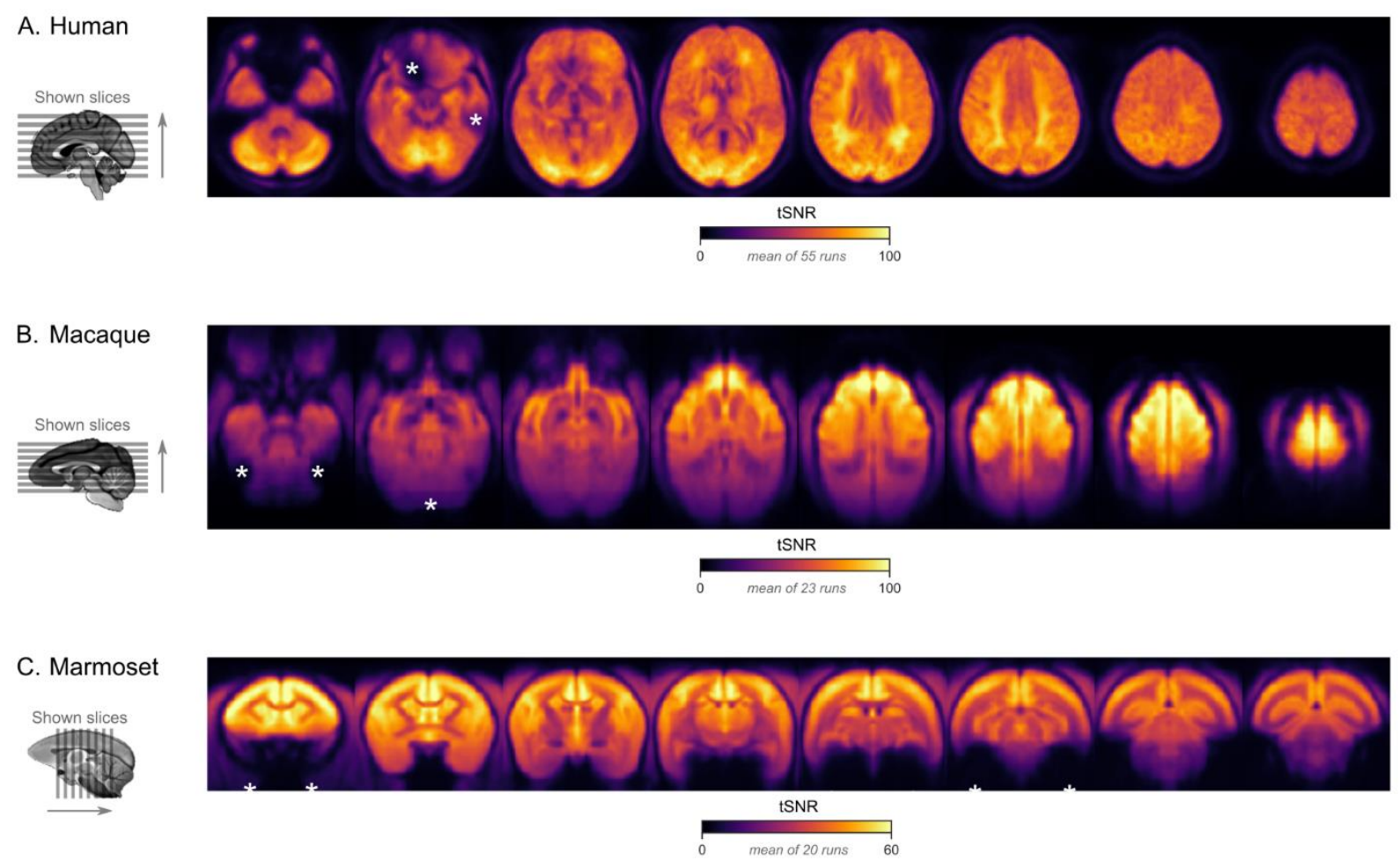

D. Rat
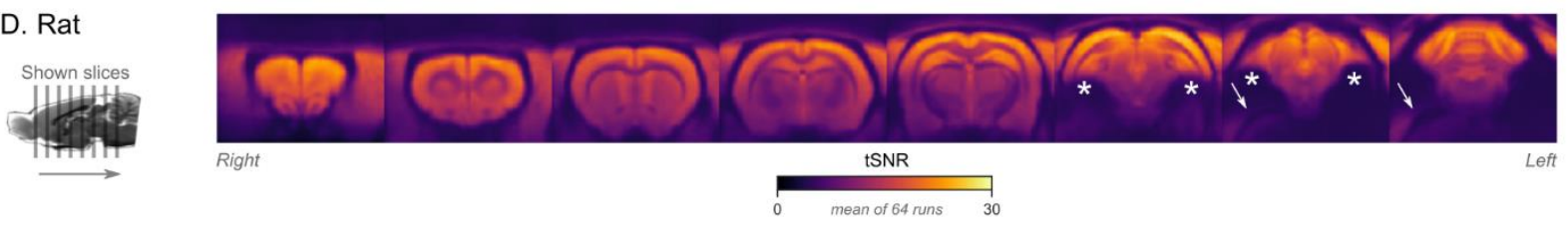

Figure S5. Temporal signal-to-noise (tSNR) maps across species. The tSNR maps - (A) human, (B) macaque, (C) marmoset, (D) rat - are overlaid on species-specific volumetric templates (same slices as in Figs. 2, 3, 5, and 6). For each fMRI run, tSNR was computed prior to preprocessing by dividing the mean of the EPI image across time with its standard deviation across time. The maps shown here represent the average tSNR values across all analyzed fMRI runs (with or without burst-suppression). Note the different scale bars for each species, indicating an overall reduction of tSNR in the smaller animals. Asterisks indicate areas with low tSNR, either due to increased distance from the coil (e.g. cerebellum in macaques), or due to susceptibility-induced distortions and signal drop-outs (e.g. orbitofrontal cortex in humans, medial temporal regions in marmosets, areas close to the ear canals in rats). An EPI ghost artifact is indicated by arrows on the rat map. 
A. Seed areas BOLD (\%)

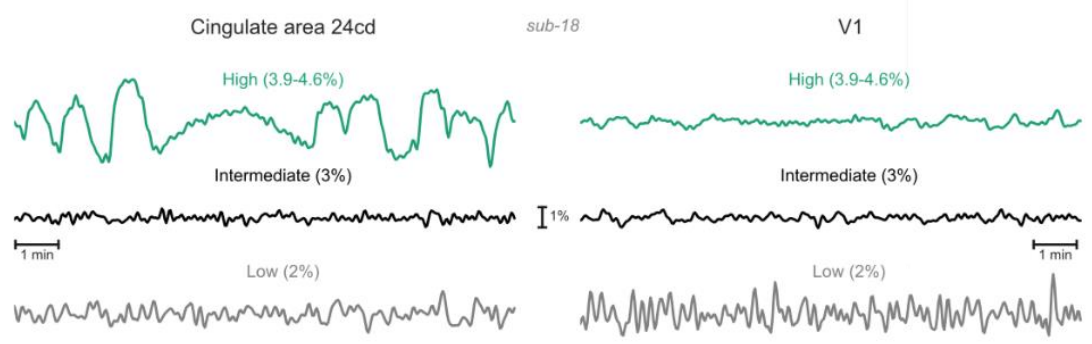

C. Power Spectral Density (PSD)

Cingulate area $24 \mathrm{~cd}$

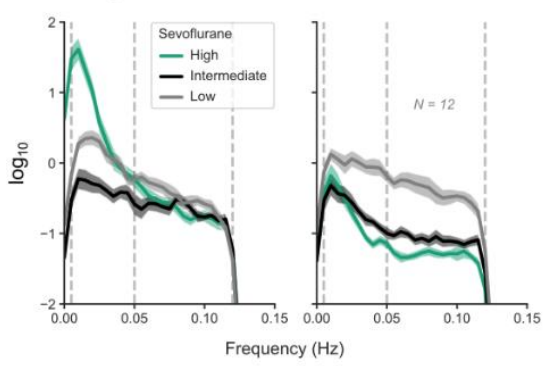

D. PSD $0.005-0.05 \mathrm{~Hz}$

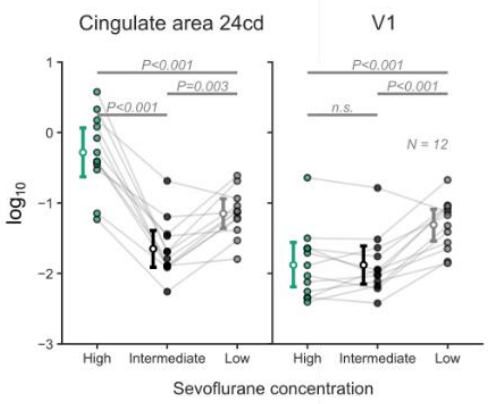

B. BOLD SD (\%)

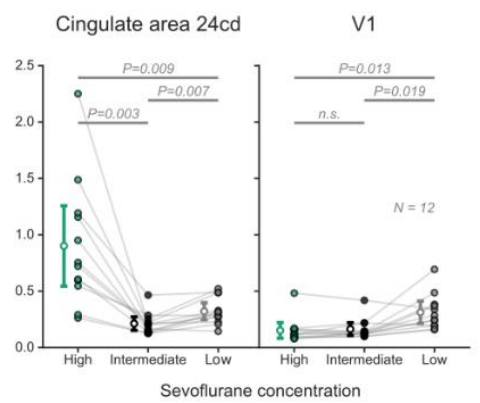

E. PSD $0.05-0.12 \mathrm{~Hz}$

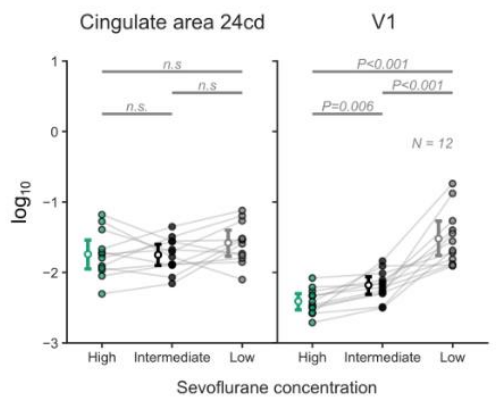

Figure S6. BOLD signal time series across sevoflurane concentrations in humans. (A) BOLD signal time series extracted from two regions-of-interest-left cingulate area $24 \mathrm{~cd}$ and left $\mathrm{V} 1$ - of a human subject, across three concentrations of sevoflurane anesthesia. (B) The standard deviations (SD) of these time series are plotted as dots across 12 human subjects. The estimated Least Squares Means, with 95\% confidence intervals, are plotted to the left of the dots. $P$ values indicate the results of Tukey post-hoc pairwise comparisons, following a repeated-measure ANOVA (sevoflurane concentration as the within-subject repeated measure). (C) For each region and sevoflurane concentration, the decadic logarithm of the BOLD signal's power spectral density (PSD) is plotted as mean \pm s.e.m. across the 12 subjects. (D) Integrated $\log _{10} \mathrm{PSD}$ within a low $(0.005-0.05 \mathrm{~Hz})$ frequency range. (E) Integrated $\log _{10} \mathrm{PSD}$ within a high $(0.05-0.12 \mathrm{~Hz})$ frequency range. Estimated Least Squares Means, confidence intervals, and $P$ values are shown as in panel B. N: number of subjects; n.s.: nonsignificant. Detailed results of repeated-measures ANOVAs and post-hoc tests are given in Tables S1 and S2, respectively. 


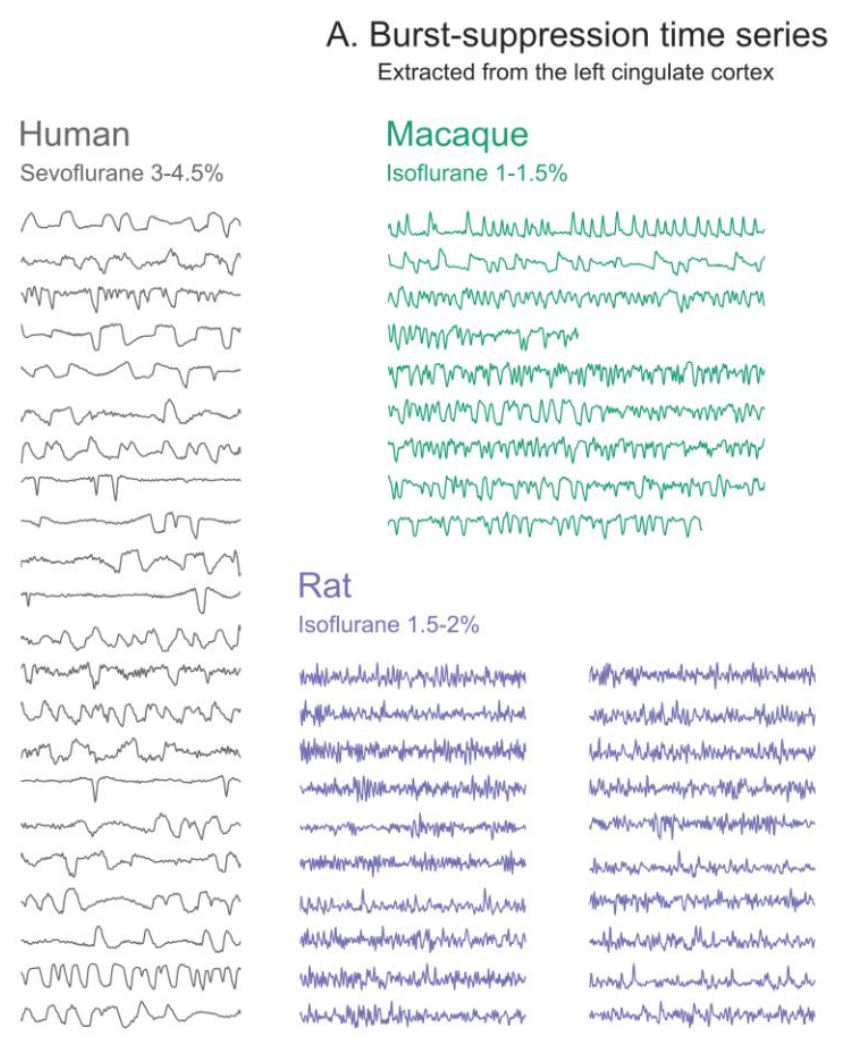

\section{B. Power Spectral Density \\ mean \pm s.e.m}

Marmoset

Isoflurane $1-1.5 \%$

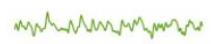

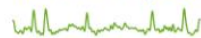

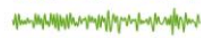

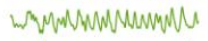

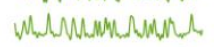

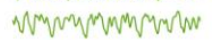

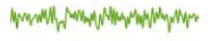

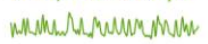
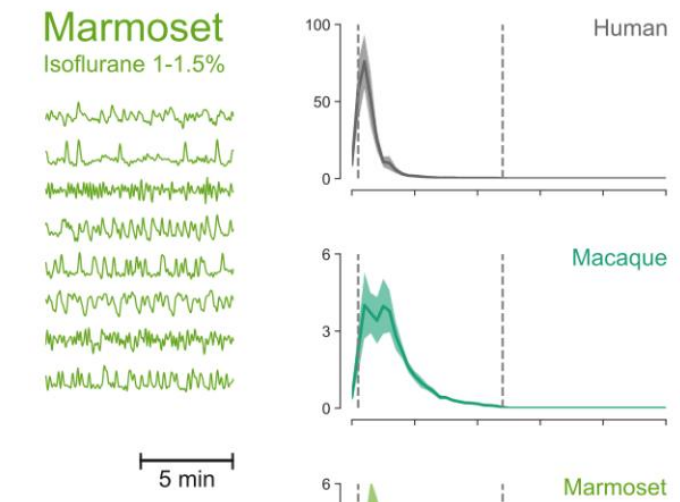

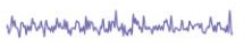

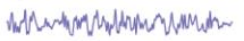

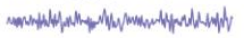

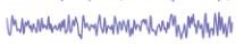

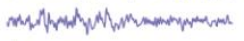

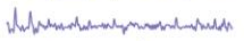

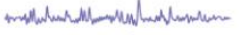

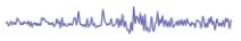

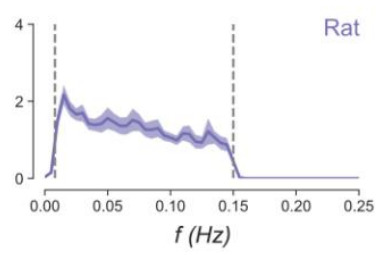

Figure S7. Burst-suppression timescales across species. (A) BOLD fMRI signal time series extracted from a region-of-interest in the cingulate cortex are shown for all runs classified as burst-suppression across the four species. This region-also used for the seed-based analysis (see Fig. 8) - was selected because of its strong correlation with burst-suppression (i.e. asymmetric PCs) in all species. All time series are shown here min-max scaled (between 0 and 1) to put the emphasis on differences in time, not in amplitude. The time series peaks appear shorter in duration and occur more frequently as we move from humans to nonhuman primates and finally, to rats. (B) This effect is reflected on the power spectral density plots of the time series, with increasingly higher frequencies being present from top to bottom. Power spectral density was computed from time series normalized to percent-signal-change (relative to the mean), prior to min-max scaling. Vertical dashed lines indicate the cutoffs of the bandpass filter that was used for fMRI preprocessing. 


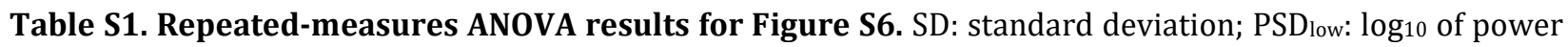
spectral density integrated between $0.005-0.05 \mathrm{~Hz}$; $\mathrm{PSD}_{\text {high: }} \log _{10}$ of power spectral density integrated between 0.05-0.12 Hz; Cg: left cingulate area 24cd; V1: left primary visual cortex; GG: Greenhouse-Geisser.

\begin{tabular}{lllllllll} 
Variable & Effect & $\begin{array}{l}\text { Sphericity } \\
\text { correction }\end{array}$ & $\begin{array}{l}\text { Sum of } \\
\text { Squares }\end{array}$ & df & $\begin{array}{l}\text { Mean } \\
\text { Square }\end{array}$ & F & p & $\boldsymbol{\eta}^{2}$ \\
\hline SD in Cg & Dose & GG & 3.29 & 1.05 & 3.1310 & 16.5 & 0.002 & 0.470 \\
& Residual & GG & 2.20 & 11.56 & 0.1900 & & & \\
SD in V1 & Dose & GG & 0.194 & 1.13 & 0.1723 & 11 & 0.005 & 0.285 \\
& Residual & GG & 0.194 & 12.38 & 0.0157 & & & \\
PSD $_{\text {low in } \text { Cg }}$ & Dose & None & 11.51 & 2 & 5.7560 & 46.3 & $<0.001$ & 0.646 \\
& Residual & None & 2.74 & 22 & 0.1240 & & & \\
PSD $_{\text {low in V1 }}$ & Dose & None & 2.56 & 2 & 1.2785 & 23.2 & $<0.001$ & 0.293 \\
& Residual & None & 1.21 & 22 & 0.0552 & & & \\
PSD $_{\text {high in }}$ Cg & Dose & None & 0.216 & 2 & 0.1082 & 1.82 & 0.186 & 0.073 \\
& Residual & None & 1.308 & 22 & 0.0595 & & & \\
PSD $_{\text {high in V1 }}$ & Dose & GG & 5.22 & 1.28 & 4.0790 & 36.2 & $<0.001$ & 0.677 \\
& Residual & GG & 1.58 & 14.07 & 0.1130 & & & \\
\hline
\end{tabular}

Table S2. Post-hoc pairwise tests for Figure S6. SD: standard deviation; PSD $D_{\text {low: }} \log _{10}$ of power spectral density integrated between $0.005-0.05 \mathrm{~Hz}$; PSD high: $\log _{10}$ of power spectral density integrated between $0.05-$ $0.12 \mathrm{~Hz}$; Cg: left cingulate area 24cd; V1: left primary visual cortex.

\begin{tabular}{lllllll} 
Variable & Comparison & Mean Difference & SE & df & t & $\mathbf{p}_{\text {tukey }}$ \\
\hline SD in Cg & High - Intermediate & 0.689 & 0.1571 & 11 & 4.39 & 0.003 \\
& High - Low & 0.580 & 0.1565 & 11 & 3.70 & 0.009 \\
& Intermediate - Low & -0.109 & 0.0287 & 11 & -3.89 & 0.007 \\
SD in V1 & High - Intermediate & -0.0119 & 0.0132 & 11 & -0.905 & 0.649 \\
& High - Low & -0.1613 & 0.0464 & 11 & -3.472 & 0.013 \\
& Intermediate - Low & -0.1493 & 0.0455 & 11 & -3.280 & 0.019 \\
PSD ${ }_{\text {low }}$ in Cg & High - Intermediate & 1.369 & 0.1560 & 11 & 8.79 & $<0.001$ \\
& High - Low & 0.868 & 0.1590 & 11 & 5.48 & $<0.001$ \\
& Intermediate - Low & -0.501 & 0.1130 & 11 & -4.42 & 0.003 \\
PSD $_{\text {low in V1 }}$ & High - Intermediate & 0.0029 & 0.0666 & 11 & 0.0436 & 0.999 \\
& High - Low & -0.5639 & 0.1086 & 11 & -5.1917 & $<0.001$ \\
& Intermediate - Low & -0.5668 & 0.1066 & 11 & -5.3177 & $<0.001$ \\
PSD $_{\text {high in V1 }}$ & High - Intermediate & -0.228 & 0.0574 & 11 & -3.98 & 0.006 \\
& High - Low & -0.897 & 0.1355 & 11 & -6.62 & $<0.001$ \\
& Intermediate - Low & -0.669 & 0.1198 & 11 & -5.58 & $<0.001$ \\
\hline
\end{tabular}

